\title{
La notion de "régime d'assemblée » et les origines de la classification des régimes politiques
}

ARN AUD LE PILLOUER

Le débat doctrinal relatif à la réduction du mandat présidentiel à cinq ans en octobre $2000^{1}$ a fait une large place à la question de la « nature » du régime $e^{2}$. Pourtant, la classification traditionnelle des régimes politiques (qui distingue les régimes parlementaires et présidentiels) est l'objet depuis de nombreuses années de critiques qui pouvaient sembler lui avoir été fatales ${ }^{3}$. II avait été démontré non seulement que ces catégories ne s'opposai ent pas, mais qu'en outre, ranger tel ou tel régime concret sous I'une quel conque de ces classes ne permettait pas d'en tirer de conclusions quant à son fonctionnement concret ${ }^{4}$. Le fait que les spécialistes de droit constitutionnel ${ }^{5}$, comme d'ailleurs les

Arnaud Le Pillouer, ATER à I'U niversité de Cergy-Pontoise.

1. Loi constitutionnelle $n^{\circ} 2000-964$ du 2 octobre 2000 relative à la durée du mandat du président de la République, J ournal officiel de la République française, 3 octobre 2000, p. 15582.

2. L'une des questions les plus disputées, au cours de ce débat public, fut celle de savoir si la réforme instituant le quinquennat allait ou non orienter le système politique français vers le " régime présidentiel ».

3. Ces critiques ont été portées de toutes parts : d'un point de vue politiste, on a pu souligner combien était inoperante une classification exclusivement fondée sur l'existence (ou l'absence) de certaines règles constitutionnelles; du point de vue de la théorie du droit, on a insisté davantage sur les faiblesses structurelles de la classification. Pour s'en tenir aux constitutionnalistes, on peut par exemple citer Georges Burdeau (T raité de science politique; t. V : L es régimes politiques, Paris, LGDI, 2e éd., $1970, \mathrm{p}$. 385 et 441 et $\mathrm{S}$.), comme représentant du premier point de vue (cf. Francis $\mathrm{H}$ amon, « La théorie des régimes politiques dans l'œuvre de Georges Burdeau », in Le pouvoir et l'Etat dans l'œanvre de G eorges B urdeau, Paris, PUAM, « Droit public positif », Economica, 1993, p. 59 à 71), et Charles Eisenmann comme représentant du second (C. Eisenmann, "Essai de classification théorique des formes politiques ", Politique - Revue internationale des idées, des institutions et des événements politiques, $\mathrm{N}$ ouv. série, $\mathrm{n}^{\circ} 41-44$, Paris, CN RS, 1968, p. 5 à 86).

4. C'est ce que C. Eisenmann a appelé (op. cit., p. 10) les valeurs « logique » et « scientifique $\gg$ de la classification.

5. Cf. Revue du droit public, n 4-2000, p. 943 et s. L'ouvrage d'Olivier Duhamel sur la question ( $L$ e Quinquennat, Paris, Presses de sciences Po, 2000, p. 55 et s.) porte égal ement témoignage de l'importance des arguments fondés sur cette classification.

R evue française de D roit constitutionne, 58, 2004 
hommes politiques ${ }^{6}$, aient néanmoins tenu, au cours du débat relatif au quinquennat, à user largement d'arguments fondés sur cette classification n'est pour autant guère surprenant. Les catégories de régimes politiques qu'elle distingue sont en effet d'une grande utilité rhétorique pour celui qui les emploie : qualifier un régime de « parlementaire » ou de « présidentiel » permet en fait de formuler des prescriptions implicites - en raison du réal isme ontologique propre au discours doctrinal ${ }^{7}$ - relativement aux réformes qu'il convient (ou pas) d'y mener.

Cependant, même si les critiques adressées à cette classification semblent n'avoir pas eu l'effet escompté, il convient de reconnaître qu'elles n'ont pas non plus été sérieusement contestées. Aussi est-il inutile de revenir ici sur leur démonstration. Le débat sur le quinquennat n'aura toutefois pas eu le seul mérite de mettre à nouveau en évidence, s'il en était besoin, la prégnance de l'opposition entre régimes parlementaires et régimes présidentiels dans le discours doctrinal contemporain. II aura égal ement permis de voir réapparaître une notion que l'on aurait pu croire abandonnée : celle de "régime d'assemblée ». Cette catégorie continuait certes d'être mentionnée par la plupart des manuels de droit constitutionnel parmi les grandes classes de régimes politiques 8 . Cependant, non seulement son évocation se faisait de plus en plus brève, mais cette notion avait surtout complètement disparu du débat public - qu'il soit politique ou doctrinal - depuis plusieurs dizaines d'années. La mention du concept de " régime d'assemblée » lors du débat relatif au quinquennat confirme du reste cette impression d'abandon, puisqu'il n'y apparaît que par une sorte de ricochet : les auteurs qui le mentionnent ne font en réalité que reprendre à leur compte une argumentation dirigée contre l'institution du quinquennat, qui avait été avancée en 1971 par Georges Pompidou, alors Président de la République?. Celle-ci consistait à affirmer que si le mandat présidentiel était calqué sur celui des députés, cela impliquerait un retour au « régime des partis » - autrement dit au « régime d'assemblée » qui avait prévalu sous les

6. Cf. R evue pol itique et parlementaire, $n^{\circ} 1006$, mai/juin 2000, «Q Quinquennat : réforme ou VIe République? », p. 17 et S.

7. Sur le réalisme ontologique qui caractérise les classifications juridiques, on peut se reporter à l'étude de Michel Troper, "Les classifications en droit constitutionnel », in Pour une théorie juridique de l'É tat, Paris, PUF, « Léviathan », 1994, p. 259-260.

8. Cf. infra.

9. C'est ce que rapportent Christophe Boutin et Frédéric Rouvillois, Quinquennat ou septennat ?, Paris, Flammarion, "D ominos », 2000. Évoquant les véritables menaces que fait (sel on eux) peser sur le régime de la $V$ e République la réforme sur le quinquennat, il's aj outent en effet : « En 1971 déjà (...) le President Pompidou écrivait à l'un de ses intimes l'écrivain Roger Ikor: " Je ne suis pas pour le mandat de cinq ans, qui conduit fatal ement à une convergence des élections présidentielle et législatives, et donc au régime présidentie inté gral, ou au régime d'assemblée ». Tels sont, en effet, les deux prolongements possibles, et antinomiques, de l'instauration du quinquennat : le régime présidentiel ou le régime d'assemblée » (ibid., p. 93). 
IIIe et IV e Républiques ${ }^{10}$. L'argument, qui semble a priori peu convaincant $^{11}$, paraît surtout foncièrement suranné1 ${ }^{12}$. A ussi I'usage de la notion de « régime d'assemblée » dans ce débat confirmet-il, paradoxalement, sa quasi-disparition du champ conceptuel de la doctrine constitutionnelle contemporaine. Or, c'est cette désuétude qui, précisément, suscite les interrogations.

II convient en effet de rappeler que le « régime d'assemblée » a connu son heure de gloire - et que cette heure fut longue. II suffit de relire la doctrine classique ${ }^{13}$ pour constater que cette catégorie a toujours fait partie intégrante de la classification traditionnelle des régimes politiques. Sorte de « troisième larron » du couple régime présidentiel-régime parlementaire, elle a longtemps été présentée sur un pied d'égalité avec ces derniers et un ouvrage lui a même été entièrement consacré par l'un des plus éminents auteurs de son époque ${ }^{14}$, dans les années cinquante.

Aussi la désuétude qui frappe cette notion méritet-elle un examen plus approfondi. D'abord, parce que le "régime d'assemblée » a été, paradoxalement en raison même de cette désuétude, épargné par les objections qui ont frappé l'opposition entre régimes parlementaires et présidentiels $s^{15}$ - et que son examen critique reste donc à effectuer. Ensuite, parce que cet effacement progressif contraste de manière éclatante avec le succès persistant du binôme traditionnel. On peut dès lors espérer mieux comprendre la classification des régimes politiques ellemême, si l'on saisit les raisons pour lesquelles l'une de ses composantes s'est effacée, tandis que les autres ont subsisté. À cet égard, I'hypothèse souvent avancée et selon laquelle cette catégorie serait sur le déclin en

10. Ainsi, dans la revue R egards sur l'actualité ( $n^{\circ} 266$, nov.-déc. 2000, Paris, La Documentation française, 2000), Christian Bigaut résume les arguments en défaveur du quinquennat. Sel on lui, I'un d'entre eux consiste à affirmer que la concomitance des élections présidentielles et parlementaires conduira à ce que « le candidat à la présidence verra son sort lié à celui des députés - et vice-versa - de façon immédiate et spectaculaire; le président sera l'otage des partis de sa majorité, I'obligé des parlementaires; il n'aura plus vis-à vis d'eux l'independance nécessaire et pourrait ne plus apparaître comme le représentant de la N ation mais comme le mandataire d'une de ses fractions; il sera partie prenante des guérillas parlementaires. Ce sera le retour au régime d'assemblées » (ibid., p. 14).

11. On peine véritablement à croire qu'il ait convaincu ceux-là memes qui I'utilisent, tant le danger de voir la V e République s'orienter vers un « régime d'assemblée » parait loin de se réaliser.

12. L'expression de " régime des partis », très gaullienne, accentue du reste cette impression.

13. Ainsi peut-on citer, entre autres, Adhémar Esmein, Éléments de droit constitutionned, Paris, Larose, 1896 ; Léon 'D uquit, M anuel de droit constitutionne, Paris, Fontemoing, 1903; J oseph Barthélémy et Paul Duez, T raité de droit constitutionne, Paris, Dalloz, 1933 ; Maurice H auriou, Précis élementaire de droit constitutionne, Paris, Sirey, 4 e éd. 1938 ; Julien Laferrière, $M$ anud de droit constitutionne, Paris, D omat-M ontchrestien, 2 éd. 1947, etc.

14. Paul Bastid, Le gouvernement d'assemblée, Paris, Cujas, 1956.

15. Ainsi Richard Moulin (L e présidentialisme et la classification des régimes politiques, Paris, LGDJ, 1978, p. 10, note 6) et M. Troper (op. cit., p. 252), par exemple, ne font que mentionner ce troisième terme, pour concentrer leurs critiques sur l'opposition fondamentale entre régimes parlementaire et présidentiel. 
raison de la disparition des systèmes politiques concrets qui la constituent ${ }^{16}$, ne peut être retenue : d'une part en effet, la classe des régimes d'assemblée n'a jamais été très riche - et la situation n'a donc guère évolué; d'autre part, la catégorie des "régimes présidentiels», par exemple, n'est à cet égard pas beaucoup mieux lotie, et I'on n'annonce pas pour autant sa disparition prochaine.

Du reste, si l'on espère comprendre pourquoi cette notion est de moins en moins utilisée, il convient de saisir égal ement, et à l'inverse, les raisons de son succès passé - c'est-à-dire, en définitive, de révéler la fonction que cette notion a pu revêtir dans le discours doctrinal. Encore fautil, pour être en mesure d'analyser cette notion, en déterminer la signification. II ne s'agit bien sûr pas d'exprimer ici ce qu'est réellement un " régime d'assemblée » (c'est là le travail que s'assigne général ement la doctrine), mais de déterminer les cas où la doctrine use de cette notion ${ }^{17}$. Peu importe al ors que l'auteur concerné utilise cette expression (" régime

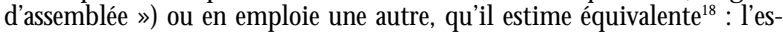
sentiel est que le concept soit bien le même. Or, pour déterminer ces cas, il est nécessaire d'avoir recours à la stipulation. On estimera donc ici que l'on est en présence de la notion de "régime d'assemblée » Iorsqu'un auteur, d'une part, évoque une catégorie de régimes distincte des régimes parlementaire et présidentiel et, d'autre part, distingue cette catégorie des deux précédentes notamment par le fait que l'A ssemblée législative y occupe une place prépondérante par rapport aux autres organe ${ }^{19}$.

II convient en outre de remarquer que cette définition n'est satisfaisante que parce que l'on adopte ici un point de vue exclusivement métathéorique. Elle serait au contraire très insuffisante d'un point de vue strictement théorique - c'est-à-dire si l'on entendait I'utiliser pour classer des régimes concrets dans ou hors de cette catégorie. Du reste, si les auteurs estiment unanimement que le « régime d'assemblée » se caractérise bien par la place prépondérante qu'y occupe l'organe législatif, ils se divisent en revanche très nettement quant à la question de savoir ce que cette "domination » signifie. En d'autres termes, la définition du « régime d'assemblée » fait l'objet de divergences fondamentales au sein

16. J ean-Louis Quermonne explique quant à lui, pour l'éliminer de ses descriptions, que le régime d'assemblée est « aujourd'hui récusé pour son "ingouvernabilité" 》 (.-L. Quermonne Les régimes politiques occidentaux, Paris " Points » Seuil 4e éd. 2000, p. 155). On pourrait lui objecter que le " régime présidentiel » s'est également vu opposer ce type d'arguments.

17. En d'autres termes, nous entendons mener ici une analyse métathéorique (et non théorique) sur la notion de "régime d'assemblée ».

18. Les expressions " régime d'assemblée », " gouvernement d'assemblée », " régime conventionnel » ou " régime directorial » sont parfois employées l'une pour l'autre, al ors qu'elles peuvent, dans d'autres contextes, revêtir des significations très différentes.

19. Cette stipulation est fondée sur le constat que tous les auteurs qui emploient l'expression " régime d'assemblée » le font en ce sens minimal : ils divergent ensuite (on le verra) sur la signification, les modalités ou bien encore les conséquences de cette prépondé rance législative. 
de la doctrine constitutionnelle, au point que la notion elle-même finit par paraitre manquer de substance (I). C'est pourquoi il convient d'examiner les conditions dans lesquelles le " régime d'assemblée » a vu le jour en tant que catégorie de régimes politiques (II) : saisir le mystère de son apparition devrait en effet permettre de préciser la fonction que cette notion a pu remplir dans le discours doctrinal - et, par conséquent, les raisons de sa désuétude (III).

\section{I - L'INDÉTERMINATION DE LA NOTION DE «RÉGIME D'ASSEMBLÉE »}

Parcourir la littérature constitutionnelle à la recherche d'une définition du « régime d'assemblée », peut s'avérer quelque peu frustrant. Le chercheur est en effet rapidement déconcerté par I'hétérogénéité des classements opérés par les auteurs. De fait, les divergences relatives aux régimes concrets qu'il convient de classer dans cette catégorie de régimes sont très profondes. Elles résultent simplement du fait que le consensus autour de la définition du "régime d'assemblée »- présenté comme une catégorie de régimes dans laquelle l'organe législatif domine le système institutionnel - n'est en réalité que de façade : I'entente superficielle existant entre les auteurs tient seulement au caractère vague des termes choisis $5^{20}$. On est à l'inverse frappé, à la lecture des définitions proposées par les auteurs, par la diversité des critères mobilisés (1). Les divergences sont en réalité telles que se pose la question de l'utilité même, pour la doctrine, de disposer d'un tel concept (2).

\section{1 - LA DISPARITÉ DES DÉFINITIONSDE LA NOTION}

La disparité des définitions se concrétise surtout, dans les analyses doctrinales, par la profonde hétérogénéité des classements opérés par les auteurs. Deux positions extrêmes, mais symptomatiques, peuvent illustrer cette diversité. Sel on la première, presque tous les régimes habituellement qualifiés de " parlementaires » devraient en réalité être classés parmi les « régimes d'assemblée ${ }^{21}$; sel on la seconde, seule la Conven-

20. P. Bastid reconnaissait que les auteurs, dans leurs tentatives de définition du « régime d'assemblée », s'en tenaient pour l'essentiel «à des notions plus ou moins vagues de domination ou de prépondérance du corps législatif » (L e gouvernement d'assemblée, op. cit., p. 1). 21. Bernard Chantebout (D roit constitutionnel et science politique, Paris, Armand 'Colin, $17^{e}$ éd., 2000, p. 244) explique en effet : " Final ement, à l'exception de quelques vestiges hérités du parlementarisme classique - telles la présence à la tête du pays d'un chef de l'État, désormais sans pouvoirs, la pratique de la " question de confiance », inefficace et contestée, ou l'affirmation purement théorique du principe de solidarité ministérielle - rien ne distingue vraiment le régime parlementaire moniste du régime d'A ssemblée ». La res- 
tion nationale de 1792 aurait véritablement réal isé ce type de régime, les autres ne méritant pas cette qualification ${ }^{22}$. II est remarquable que la première fasse disparaître la catégorie des « régimes parlementaires » en l'englobant dans celle, plus vaste, des " régimes d'assemblée ${ }^{23}$, tandis que la seconde entraîne au contraire l'anéantissement de cette dernière classe, puisqu'un seul objet peut y être rangée ${ }^{24}$.

Entre ces deux thèses radicales, on trouve au sein de la doctrine toutes sortes de positions, des plus restrictives aux plus extensives quant à l'ampleur de la catégorie des "régimes d'assemblée ». M is à part le régime institué par la Convention nationale de 1792 durant son mandat ${ }^{25}$ - peutêtre d'ailleurs parce que cette dernière a donné son nom à un synonyme de l'expression " régime d'assemblée »-, aucun système politique concret ne fait réellement I'unanimité. M ême la Suisse, que I'on cite parfois comme le véritable modèle du "régime d'assemblée », au même titre que la Grande-Bretagne et les États-U nis pour les régimes parlementaire et présidentiel ${ }^{26}$, est exclue de cette catégorie de régimes par un nombre

triction introduite par l'auteur, selon laquelle seuls les régimes parlementaires monistes seraient concernés par cette " re-qualification », ne doit pas tromper : dans la mesure où tous les régimes aujourd'hui qualifiés de "parlementaires », sont monistes, et où tous ceux qui seraient susceptibles d'être dits " dualistes » - tel le régime de la Ve République, par exemple - sont en général exclus de cette catégorie, son propos vise bien à qual ifier tous les régimes habituel lement désignés comme parlementaires, de " régimes d'assemblée ». C'est du reste ce que Bernard Chantebout confirmait lui-même dans l'article qu'il avait consacré à cette question ("Le régime parlementaire moniste, gouvernement d'assemblée », in M élanges offerts à G eorges B urdeau - L e pouvoir, Paris, LGDJ, 1977, p. 43) : " au régime parlementaire classique que nous n'hésiterons pas à qualifier de seul authentique, a succédé partout, à la fin du $X I x^{e}$ siècle ou au début du $x x^{\mathrm{e}}$ selon les États, un régime parlementaire communé ment appelé « moniste » qui, certes, donne lieu à des pratiques très différentes en fonction des traditions nationales, mais repose néanmoins sur des principes communs, lesquels l'apparentent en fait au régime d'assemblée ».

22. Pierre Pactet, Institutions pol itiques - D roit constitutionnel, Paris, Armand Colin, 19e éd. 2000. L'auteur indique en effet : " Le seul exemple véritablement probant est celui du régime Conventionnel (1792-1795), mais il appartient au passé » (ibid., note 1, p. 142). $C$ 'est semblet-il égal ement la position de Claude Leclercq ( $D$ roit constitutionnel et institutions politiques, Paris, Litec, 7e éd., 1990, p. 216-217).

23. C'est bien là l'opinion de $B$. Chantebout («Le régime parlementaire moniste, gouvernement d'assemblée », op. cit., p. 45) : " Ce qui se dissimule à présent sous cette appellation (de « parlementarisme »), c'est le régime d'assemblée ».

24. C'est ainsi que P. Pactet explique: « C'est une catégorie que I'on fait traditionnelle ment figurer aupres des deux précédentes mais de manière un peu abusive car on ne peut guère citer d'exemples contemporains véritablement typiques » (ibid., p. 142). Quant à C. Leclercq, l'intitulé du paragraphe qu'il consacre à cette catégorie (« Un mythe, une déviation ou une utopie : le régime d'assemblée », op. at., p. 216) est suffisamment significatif.

25 . Toutefois, certains auteurs semblent vouloir réserver le qualificatif de "régime d'assemblée », sous la Convention, à celui qui eut cours avant et après la " dictature de Robespierre ». Sel on eux, durant cet intervalle, c'est le Comité de salut public qui a " exercé une véritable dictature de fait sur I'A ssemblée, et non l'inverse » (C. Leclercq, op. cit., p. 217).

26. Dans la classification des régimes politiques proposée par Marcel Prélot et Jean Boulouis (Institutions politiques et droit constitutionne, Paris, Précis Dalloz, 10 éd., 1987, p. 92 93), chacun des régimes parlementaire, présidentiel et conventionnel est associé à un système politique concret lui servant de " modèle » : la Grande-Bretagne en fait office pour le régime parlementaire et les Etats-Unis pour le régime présidentiel, tandis qu'en ce qui concerne le régime conventionnel, il est précisé que « concrètement, le gouvernement suisse 
de plus en plus important d'auteurs ${ }^{27}$. Les auteurs semblent d'ailleurs cruellement manquer d'exemples, même historiques, en la matière ${ }^{28}$.

Ils semblent éprouver moins de difficultés à citer des applications de " régimes d'assemblée » au cours de l'histoire constitutionnelle fran-

sert cette fois de référence » (ibid., p. 92). Dans le même sens, on peut également citer Julien Laferrière (op. cit., p. 750-751), René Capitant ("Régimes parlementaires ", in M élanges C arréde M alberg, Paris, Recueil Sirey, 1933, p. 51) ou Léon Duguit (M anuel dedroit constitutionned, op. at., p. 385), quoique ce dernier n'emploie pas l'expression de " régime d'assemblée » (il ne fait usage que du concept, cf. supra).

27. Ce paraît même être devenu la thèse la plus répandue aujourd'hui. Ainsi Philippe Ardant note-t-il que « la Suisse, référence classique dans ce domaine, n'est pas véritablement un régime d'assemblée » (P. A rdant, Institutions politiques et droit constitutionne, Paris, LGDJ, 12 éd., 2000, p. 340). P. Pactet explique que "le regime politique suisse a parfois été présenté comme un régime d'Assemblée (... ). Mais cette analyse ne paraît pas exacte » (P. Pactet, op. cit., p. 154). Plus radical ement encore, C. Leclercq estime à ce propos (op. cit., p. 217) que « bien loin de constituer un régime d'assemblée, il s'agirait en fait d'un régime directorial où le Conseil fédéral exerce l'essentiel des pouvoirs dans l'État ». De la même manière, J acques Cadart estime (Institutions pol itiques et droit constitutionnel, Paris, LGDJ, 1975, $\mathrm{t}$. régime d'assemblée » est "indé fendable aujourd'hui comme hier, et il est surprenant qu'une telle théorie puisse survivre aussi longtemps devant le spectacle que donne le régime suisse ». Quelques manuels de droit constitutionnel se contentent d'indiquer qu'il est « préférable » de parler, pour la Suisse, de « régime directorial » (Charles Debbasch, J ean-M arie Pontier, Jacques Bourdon et J ean-Claude Ricci, D roit constitutionnd et institutions politiques, Paris, Economica, 3e éd. 1990 , p. 156). Ce classement est d'ailleurs repris par l'essentiel des « dictionnaires de droit constitutionnel » (par ex. Pierre A vril, J ean Gicquel, D roit constitutionnel, Paris, PUF, coll.

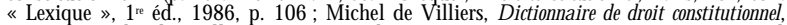
Paris, A rmand Colin, coll. "Cursus », 2e éd., 1999, p. 185-186 ; D ictionnaire constitutionne, dir. O. Duhamel et Y. Mény, Paris, Puf, 1992, article " Régime d'assemblée ", signé J. Gicquel, p. 877). Sur ce point, la thèse de J. Laferrière sel on laquelle mal gré le caractêre collégial des organes exécutifs institués par les constitutions suisse et de l'an III, " il ne paraît pas possible de ranger dans une catégorie commune des systèmes qui, comme ceux de la Suisse et de la Constitution de I'an III, procèdent de conceptions directement opposées quant à la situation de l'Exécutif par rapport au Parlement » J. Laferrière, op. cit., note 1, p. 750), semble être franchement abandonnée. II est vrai que lorsque la Suisse était classée par la majorité des auteurs parmi les régimes d'assemblée, il était fréquent que quelques nuances soient apportées à ce classement : dans cette doctrine « classique », on indique par exemple que si, " théoriquement », la Constitution helvétique organise bien un gouvernement d'assemblée, "le fait est tout différent » (J. Barthélémy et P. Duez, op. cit., p. 148 et s.), ou bien l'on invoque les traditions culturelles et l'esprit de sagesse suisses pour expliquer le fonctionnement harmonieux, dans ce pays, d'un système que l'on venait de présenter comme impraticable (A. Esmein, Eléments de droit constitutionne, 1896, p. 318).

28. $P$. A rdant reconnaît d'ailleurs explicitement que « le régime conventionnel est une forme relativement peu répandue de régime politique. (...) Dans les sociétés contemporaines, il n'existe que peu d'exemples d'un vrai régime d'assemblée » (op. cit., p. 340). $C$. Leclercq écrit aussi que « le régime d'assemblée n'a pratiquement jamais fonctionné en Europe occidentale » (op. cit., p. 217). Si le Long Parlement anglais recueille quel ques citations (P. Bastid, op. cit., p. 37 a 127 ; M. Prélot et J. Boulouis, op. cit., p. 101 ; J. Cadart, op. cit., t. I, p. 401-402), on voit surtout apparaitre dans les manuels les plus classiques et comme exemples du régime d'assemblée, quelques constitutions de l'entre-deux guerres dans certains Etats de l'ancien Empire allemand (A. Esmein, Eléments de droit constitutionnel français et comparé, Paris, Sirey, 8e éd., 1927, notes 119, 120, 121 et 122, p. 529 ; J. Laferrière, op. cit., p. 751 ; J. Barthélémy et $P$. Duez, op. cit., p. 149-150) - quoique leur classement dans cette catégorie ne manque pas d'être à l'occasion contesté (R. Capitant, "Régimes parlementaires », op. cit., p. 50; M. Prél ot et J. Boulouis, op. dit., p. 100-101). Paul Bastid va même jusqu'à citer, dans son ouvrage éponyme, la Loi fondamentale allemande de 1949 et même la Constitution turque du 20 avril 1924, comme organisant un " gouvernement d'assemblée » (P. Bastid, op. cit., p. 371 s. et 379 s.). 
çaise, même si là encore, les divergences se multiplient entre eux. Sont parfois classés dans la catégorie des « régimes d'assemblée » ceux qui ont résulté de la pratique ou plutôt ${ }^{29}$ des dysfonctionnements des constitutions des III et IVe République ${ }^{30}$. Dans les manuels de droit constitutionnel les plus classiques, ce sont essentiellement des textes restés inappliqués qui sont cités comme des "expressions théoriques $»^{31}$ du régime d'assemblée. Les constitutions girondine $e^{32}$ et surtout montagnarde ${ }^{33}$ de 1793 sont parfois mentionnées en ce sens, de même que le projet de

29. On a toutefois pu soutenir que la Constitution du 27 octobre 1946 présentait quel ques caractères du régime d'assemblée. $P$. Bastid expliquait par exemple que « le caractêre de ce texte est (...) assez hybride »; il y trouvait « des traits qui appartiennent au gouvernement d'assemblée et des traits qui appartiennent au gouvernement parlementaire » (P. Bastid, op. cit., p. 336). Cet auteur approfondit ces idées dans un article exclusivement consacré aux « deux constitutions » de 1946 (P. Bastid : " Les deux constitutions de 1946 et le gouvernement d'assemblée ", in L'évolution du droit public - Études en I'honneur d'A chille M estre, Paris, Sirey, 1956, p. 25).

30. Ainsi P. A rdant (op. cit., p. 340) expliquet-il que « l'évolution du régime parlementaire en France sous la Constitution de la llIe puis de la IV ${ }^{\text {e }}$ République a rapproché dans les faits le régime d'un régime d'assemblée ». De même, un autre manuel (C. D ebbasch, op. cit. $\mathrm{p}$. à côté des régimes d'assemblée officiellement institués, on a parlé de régimes d'assemblée à propos de régimes parlementaires dont la pratique aboutissait à en faire des régimes d'assemblée. Le régime d'assemblée est al ors « la dégenérescence normale du régime parlementaire ». On peut appliquer la formule aux dernières années de la III R Republique et à la pratique du régime de la IV e République ». $M$. de Villiers estime égal ement (op. cit., p. 185) que « la France a connu sous les IIle et IV e Républiques cette déviation du régime parlementaire ». Leur classement parmi les régimes d'assemblée est cependant parfois contesté : ainsi $P$. Pactet expliquet-il que « la Q uatrième République qui a été souvent présentée comme un régime d'assemblée faisait sans doute une part trop importante aux organes représentatifs mais demeurait fondamentalement un régime parle mentaire, même si son fonctionnement était défectueux » (P. Pactet, op. cit., note 1, p. 142). Pour C. Leclercq (op. cit., p. 218), le classement de ces systèmes dans la catégorie des " régimes d'assemblée » relève même tout simplement "d'un abus de langage : les III et IVe Républiques ont été des régimes parlementaires simplement déséquilibrés, le déséquilibre s'exerçant en faveur du Parlement ».

31. L'expression est de J. Barthélémy et P. Duez (op cit. p. 149).

32. A. Esmein surtout évoque le projet « girondin » (Éléments... , 1896, p. 316-317) quoique avec quelques nuances - dues au caractère indépendant du Conseil exécutif dans le texte. Ce sont d'ailleurs plus les idées contenues dans le rapport de Condorcet que les articles constitutionnels qui font l'objet de ses commentaires. Le passage relatif au pouvoir exécutif qui motive ce classement est bien connu (séance du 15 février 1793, A rchives parle mentaires, t. 58, p. 591): " Ces agents (le pouvoir exécutif) doivent être essentiellement subordonnés aux dépositaires de la puissance législative, ou le principe de l'unité d'action serait violé. Ce Conseil (exécutif) doit être la main avec laquelle les législateurs agissent, l'œil avec lequel ils puissent observer les détails de l'exécution de leurs décrets et les résultats des effets que ces décrets ont produits ». I. Laferrière (op. cit. note 1, p. 749-750) mentionne égal ement, avec les mêmes nuances qu'Esmein, la « constitution girondine » comme exemple de " gouvernement d'assemblée ».

33. L. Duguit explique par exemple (M anuel de droit constitutionned, op. cit., p. 384) que la conception « d'un gouvernement républicain composé de commis, d'agents d'exécution du parlement, a trouve dans la constitution montagnarde de 1793 sa réalisation complète ». M. Prélot et J. Boulouis (op. cit., p. 100) notent égal ement que le régime d'assemblée se serait « égal ement incarné dans les constitutions de l'an I, si el le avait été appliquée ». Cette mention est d'ailleurs fréquente dans les manuels (J. Laferrière, op. cit., p. 749 ; J. Cadart, op. cit., t. I, p. 399), certains auteurs (C. Debbasch, op. cit., p. 155) allant jusqu'a affirmer que "le gouvernement d'assemblée a trouvé sa consécration en France avec la Constitution de $1793 »$ (nous soulignons). 
Constitution avorté du 19 avril 1946 ${ }^{34}$, ou le fameux amendement Grévy35, formulé en octobre 1848. Mais ce sont sans doute les régimes " transitoires » institués par les assemblées constituantes durant leur mandat qui recueillent le plus de citations ${ }^{36}$. La Convention nationale de 1792 fait l'unanimité à ce propos ${ }^{37}$, mais les assemblées nationales de 1848 et de 1871 ont également quelque succè̀ ${ }^{38}$, ainsi que, quoique à un degré moindre, le régime institué par la Loi constitutionnelle du 2 novembre 1945, « portant organisation provisoire des pouvoirs publics » durant le mandat des assemblées constituantes de 1945 et de $1946^{39}$.

34. C. Debbasch, op. cit., p. 156 (« Le projet de constitution du 19 avril 1946 tendait égal ement à établir un régime d'assemblée »); M. Prél ot et J. Boulouis, op. cit., p. 103, etc. $P$. Bastid affirme même ( $P$. Bastid, op. cit., p. 336) qu'il « y a accord général pour admettre que la constitution du 19 avril établissait un régime d'assemblée » (nous soulignons).

35. Cet amendement, rejeté par 643 voix contre 158 (sur 801 votants) lors de la séance du 7 octobre 1848 ( $M$ oniteur U niversel, p. 2749), est cité par un très grand nombre d'auteurs comme la formulation symptomatique des idées propres au « régime d'assemblée » : c'est le cas de A. Esmein (Eléments... 1896, p. 315), de L. Duguit (M anuel de droit constitutionned, op. cit., p. 384-385), de J. Barthélémy et P. Duez (op. cit., p. 149), de J. Laferrière (op. cit., p. op. cit., p. 21), de M. Prélot et J. Boulouis (op. cit., p. 100), etc. Comme presque tous les exemples de « régimes d'assemblée », le classement de l'amendement G révy sous cette catégorie ne fait toutefois pas I'unanimité: $R$. Capitant, par exemple en fait un exemple de sa " troisième variante » du régime parlementaire, celle " sans chef d'État » ("Régimes parlementaires », op. cit., p. 50-51)

36. J. Laferrière expliquait par exemple (op cit. p. 749) : «En France, cette solution fut appliquée par une sorte de nécessité par les assemblées qui, au lendemain d'une révolution, étai ent le seul pouvoir organisé. Ainsi procèdent nos assemblées constituantes ». La même remarque était d'ailleurs déià formulée par J. Barthélémy et $P$. Duez (op. cit., p. 148). Quant à ]. Cadart, il consacre une sous-partie de son exposé sur le " régime d'assemblée » aux " régimes provisoires des assemblées constituantes » (op. cit., t. I, p. 402 à 407). Leur classement dans cette catégorie n'est toutefois pas unanime, puisque sans compter les auteurs qui ne mentionnent pas tous ces régimes comme exemples de « gouvernements d'assemblée », certains auteurs réfutent à l'occasion ce classement, pour considérer certains d'entre eux comme des régimes parlementaires ( $R$. Capitant « Régimes parlementaires », $0 p$. cit., p. 50-51).

37. Aucun auteur n'omet de ranger le régime institué par la Convention nationale entre 1792 et 1795 parmi les « gouvernements d'assemblée » - la position minimaliste (cf. P. Pactet, op. cit., p. 142, note 1 , ou C. Leclercq, op. cit., p. 217) consistant à n'y ranger qu'elle. Bernard 'Chantebout dit même de la Convention qu'elle « est sans conteste l'arché type du régime d'assemblée » ( $B$. Chantebout, "Le régime parlementaire moniste, gouvernement d'assemblée », op. cit., p. 48). P. Bastid (op. cit., p. 3) indique quant à lui, dans son introduction, que c'est au régime de la Convention nationale que « tout le monde songe d'instinct ", lorsque I'on évoque le régime d'assemblée.

38. A. Esmein disait à propos du « gouvernement conventionnel » que « (c'était) lui, en effet, qui (avait) été applique en France de 1871 à 1873 » (A. Esmein, Éléments... , 1896, p. 317). L. Duguit expliquait de même (M anued de droit constitutionnd, op. cit., p. 385) qu'en «1871 on fut amené par les circonstances à organiser le gouvernement d'après cette conception ». Le manue de C. Debbasch (op. cit., p. 155) mentionne pareillement «le régime provisoire organisé par l'Assemblée nationale, entre 1871 et 1875 »; selon ce manuel, " le régime conventionnel est également le régime qu'adopte, en pratique, l'Assemblée nationale constituante de 1848 » (ibid.). C'est également l'opinion de M. H auriou (Précis élémentaire de droit constitutionnd, op. cit., p. 66). J. Laferrière (op. cit., p. 749), M. Pré lot et J. Boulouis (op. cit., p. 101), ou P. A rdant (op. cit., p. 340) citent également ces deux régimes transitoires.

39. Ce régime transitoire qui a préval u sous les deux assemblées constituantes de 1945 et de 1946 est par exemple cite, quoique de manière nuancée (cf. infra), comme une application du régime d'assemblée par M. de Villiers (op. cit., p. 185), J. Cadart (op. cit., t. I, p. 403) ou P. A rdant (op. cit., p. 340). Le cas de l'A ssemblée constituante de 1789 est quant à lui 
En réalité, il est surtout troublant de remarquer qu'à l'exception notable du système institué par la Convention nationale, il n'existe tout simplement pas de système politique concret cité comme « régime d'assemblée ", dont la qualification ne soit remise en cause au moins une fois, par un autre auteur. Ces hésitations, pour le moins saisissantes, résultent, on s'en doute, de la diversité des critères utilisés par la doctrine pour définir cette catégorie de régimes ${ }^{40}$.

Pour caractériser la domination que l'Assemblée est supposée exercer en « régime d'assemblée », les auteurs usent en effet le plus souvent de la métaphore révolutionnaire - guère éclairante - sel on laquelle l'organe exécutif, le gouvernement, est réduit au rôle de commis de l'A ssemblée ${ }^{41}$, de simple « agent » d'exécution. C'est ainsi qu'il apparait " comme une autorité subordonnée ${ }^{42}$, puisqu'il n'est en définitive que le "simple exécutant ${ }^{43}$ des volontés parlementaires. Pour peu cependant que l'on entre dans le détail de ce que la « domination » de l'assemblée sur l'exécutif signifie pour chacun de ces auteurs, ou que l'on s'interroge sur les dispositions ou les pratiques constitutionnelles qui en sont pour eux les manifestations privilégiees, cette apparente unanimité tend à s'éroder considérablement.

Même la question de savoir si ces " gouvernements d'assemblée » constituent des régimes de confusion ou de séparation des pouvoirs - distinction pourtant à la fois aussi simple que fondamentale en droit constitutionnel -, fait l'objet de discordances notoires au sein de la doctrine $^{44}$. Mais les divergences les plus décisives - parce qu'elles impli-

très rarement invoqué par les auteurs, à l'exception notable de $M$. H auriou. En raison de sa définition du " gouvernement conventionnel » comme régime dans lequel il n'y a qu'une seule Chambre, ce dernier (M. H auriou, Précis éémentaire..., op. cit., p. 65-66) classe la Constitution de 1791 elle-même dans cette catégorie de régimes: "Lá Révolution de 1789 devait créer une nouvelle forme à assemblée législative unique, très rapprochée de l'assemblée du peuple, et essayant de se subordonner entièrement le pouvoir executif et de le transformer en un simple agent d'exécution (pouvoir commis et non organe représentatif de la nation). L'assemblée unique avait été instituée par la constitution du 3 septembre 1791 et, en effet, la Constituante, la Législative et la Convention furent des assemblées uniques ». J. Cadart classe aussi, quoique de manière beaucoup plus nuancée, le système de 1789-1791 parmi les « régimes d'assemblée » (op. cit., p. 403-404).

40. P. Bastid reconnaît, dans l'avant-propos de son ouvrage éponyme (L e gouvernement d'assemblée, op. cit., p. 1), que les divergences qui existent au sein de la doctrine relativement au classement de'la Constitution de 1946 (en vigueur au moment où il écrit) comme gouvernement d'assemblée, tiennent pour l'essentiel au fait que les auteurs « ne s'entendent pas toujours sur le sens des mots. Au vrai, la plus grande confusion règne dans les conceptions relatives à cette forme politique ».

41. La liste est longue de ceux qui utilisent cette métaphore : I. Barthélémy et $P$. Duez (op. cit., p. 148); L. Duguit (M anuel..., op. cit., p. 383-384, 386 et 391); M. H auriou (Pré dis éémentaire..., op cit., p. 65) ; J. Laferriere (op. cit., p. 747) ; J. Cadart (op. cit., t. I, p. 398) M. Duverger (Institutions politiques et droit constitutionnel, Paris, PUF, "Thémis », 18e éd., 1990, t. I, p. 194); P. Ardant (op. dit., p. 339) ; C. Debbasch (op. cit., p. 154), etc.

42. P. A rdant, op. cit., p. 338.

43. C. Debbasch, op. cit., p. 154 ; C. Leclercq, op. cit., p. 216.

44. Plusieurs auteurs estiment que la domination que l'Assemblée exerce sur les autres organes a pour conséquence finale son appropriation de toutes les fonctions de l'Etat 
quent les désaccords spectaculaires observés quant au classement des régimes concrets - concernent bien sûr les critères distinctifs des " régimes d'assemblée ». II ne suffit pas en effet d'affirmer que l'assemblée y exerce une certaine domination sur le reste des organes de l'État: reste encore à déterminer en quoi consiste, concrètement, cette domination. En d'autres termes, il convient de préciser les caractéristiques propres aux « régimes d'assemblée ».

Or, on peinerait véritablement à énumérer tous les critères qui ont été, à un moment ou à un autre, utilisés pour définir cette sorte de régimes. On peut citer, à titre indicatif : I'absence de chef de l'État irresponsable ${ }^{45}$; la nomination directe des ministres par le Parlement ${ }^{46}$; le pouvoir de révocation du gouvernement dont les députés bénéficient symétriquement ${ }^{47}$ (dont les auteurs soulignent le caractère discrétion-

(L. Duguit, M anuel de droit constitutionnd, op. cit. p. 386 ; A. Esmein, Éléments de droit constitutionnd, op. cit., 1896, p. 314 ; J. Laferrière, op. cit., p. 747, etc.), certains fai sant même de cette confusion des pouvoirs ce qui distingue fondamentalement le "régime d'assemblée » des régimes parlementaire et presidentiel. Mais quelques auteurs ne caractérisent ai nsi ce type de régime que dans son « sens strict »- son « sens large » semblant échapper à cette critique (P. Ardant, op cit, p. 339 . C. Debbasch, op cit, p. 154). D'autres au contraire traitent plus ou moins explicitement du " gouvernement d'assemblée » comme d'un régime de séparation des pouvoirs ( $J$. Barthélémy et $P$. Duez, op. cit., p. 146-148), certains allant même jusqu'à qual ifier de « confusion terminologique » le fait de présenter le régime d'assemblée comme un régime de cumul des pouvoirs au profit de l'organe législatif (Georges Burdeau, Francis $\mathrm{H}$ amon et Michel Troper, D roit constitutionne, Paris, LGDJ, 26e éd., 1999, p. 98).

45. Ce critère apparaît par exemple en négatif chez A. Esmein puisque d'une part, il qualifie le régime d'assemblée de régime de confusion des pouvoirs, et que d'autre part, il explique (Eléments... , 1896, p. 311) que, si le gouvernement parlementaire respecte au contraire sel on lui lé principe de la séparation, " cela résulte d'abord de ce qu'il comporte un chef du pouvoir exécutif (roi ou président) inamovible; al ors même qu'il est nommé par le Corps législatif, il ne peut être révoqué par lui, et cela suffit pour lui assurer en droit l'indépendance qu'exige le principe de la séparation ». J. Laferrière dira plus explicitement (op. cit., p. 748) que, dans le "régime d'assemblée », « il n'y a pas de chef de l'Etat irresponsable ». Quant à J. Barthélémy et P. Duez (op. cit., p. 148), ils expliquent que « dans ce régime, l'exécutif n'est pas un pouvoir distinct en face de l'asssemblée », ce qui signifie clairement, dans le langage de l'époque, I'absence d'un chef de l'Etat indépendant des chambres législatives. Ce critère est égal ement repris, quoique de manière plus implicite, par M. H auriou (Préis éémentaire.., op. cit., p. 66).

46. Pour M. Prélot et J. Boulouis, par exemple (op. cit., p. 101) : « Le gouvernement d'Assemblée (...) résulte de l'élection du gouvernement par l'Assemblée ». J. Laferrière expliquait de la même manière que, dans ce type de régimes, "normal ement, les membres de ce conseil, ou tout au moins le chef du gouvernement, seront nommés par l'assemblée » (J. Laferrière, op. cit., p. 748). C'est d'ailleurs bien ce critère que suggérait A. Esmein, lorsqu'il expliquait que « le gouvernement parlementaire consommerait la confusion des deux pouvoirs, si les ministres, comme le voudraient quelques-uns, étaient élus en forme par la Chambre des députés. Ils cesseraient alors d'être les agents du pouvoir exécutif » (A. Esmein, Eléments... , 1896, p. 313). Ce critère est notamment repris aujourd'hui par P. Ardant (op. cit., p. 339).

47. Ainsi, J. Barthélémy et $P$. Duez (op. cit., p. 148) expliquent que dans ce système, l'exécutif « est réduit vis-à-vis de l'assemblée qui le nomme, le dirige, le révoque, au rôle de simple agent » (nous soulignons). L. Duguit indique quant à lui (M anuel... op. cit., p. 392) que le parlement, dans ce type de régimes, " doit pouvoir à chaque instant congédier (les agents du pouvoir exécutif) ». A ujourd'hui, P. Ardant, usant à nouveau de la métaphore, affirme: "l'exécutif n'est qu'une courroie de transmission à laquelle son mandat peut être retiré à tout moment » (op. cit., p. 339). 
nair $\left.\mathrm{re}^{48}\right)$; l'absence de droit de dissolution ${ }^{49}$; I'impossibilité pour le gouvernement de poser la question de confiance et, plus général ement, de démissionner ${ }^{50}$, etc. En somme, il semble que tout ce qui peut être anaIysé comme un renforcement du pouvoir du Parlement ${ }^{51}$ ou comme un affaiblissement de l'organe exécutif ait été, à un moment ou à un autre, désigné comme critère - plus ou moins fiable - du régime d'assemblée: le monocamérisme d'un côtés2 ${ }^{2}$ et la collégialité de l'organe exécutif de I'autre ${ }^{53}$, en constituent les meilleurs exemples ${ }^{54}$.

48. Les auteurs ne cessent d'insister sur le caractère discrétionnai re du droit de révocation en "régime d'assemblée », de manière notamment à mieux distinguer ce dernier du " régime parlementaire ». $P$. Bastid, par exemple, parle de « révocation ad nutum » (op, cit. p. 390), tandis que $C$. Leclercq insiste sur le fait que l'exécutif « peut, à tout moment, être démis » (op. cit., p. 217 ; nous soulignons). J. Cadart entend quant à luí souligner ce caractère discrétionnare par l'accumulation de qualificatifs : il affirme en effet (op. cit. t. I p. 398) que les agents du pouvoir exécutif sont « perpétuellement révocables au gré du seul bon plaisir de l'Assemblée».

49. L. Duguit fait par exemple remarquer ( $M$ anud... op. cit., p. 392) que dans ce type de régimes, les " agents du pouvoir exécutif » « ne peuvent exercer aucune action sur le parlement ; ills ne peuvent par exemple ni l'ajourner ni le dissoudre ». Pour P. A rdant (op. cit. p. régime d'assemblée », "il n'est pas question, bien sûr, (que l'exécutif) prononce la dissolution de l'assemblée ». Sel on I. Cadart, dans ce système, les « exécutants » n'ont pas le droit « de dissoudre la chambre, évidemment indissoluble et permanente » (op cit., t. I, p. 398). D'ailleurs, si l'on qualifie parfois les pratiques institutionnelles des IIle et IV e Républiques de "régimes d'assemblée », c'est bien, notamment, en raison de la disparition de fait du droit de dissolution dans ces régimes: le manuel de $C$. D ebbasch indique par exemple très explicitement que " cette " dégénérescence » (de ces régimes parlementaires) a pour origine l'inutilisation de certaines prérogatives, reconnues constitutionnelle ment à l'exécutif, notamment le pouvoir de dissolution de la chambre basse » (op. cit. p. 156). De même, J. Cadart indique (op. cit., t. I, p. 407) que " sous ces deux régimes, le déséquilibre des pouvoirs est sorti de l'inégalité des moyens d'action et de pression réciproques du gouvernement et du Parlement et essentiellement de l'impossibilité pour le gouvernement d'utiliser le droit de dissolution pourtant expressément prévu par la constitution ». Dans le D ictionnaire constitutionnel d'O. Duhamel et $Y$. M ény, l'article relatif au « régime d'assemblée » (rédigé par J. Gicquel) évoque également "la désuétude, voire l'abrogation, qui frappe le droit de la dissolution » (op. cit., p. 876) dans ce type de systèmes.

50. C. Leclercg, op. cit., p. 217 ; J. Cadart, op. cit., t. I, p. 398

51. Sauf les affaiblissements du pouvoir de l'assemblée qui sont censés bénéficier au peuple; le " régime d'assemblée », tel que le présentent les auteurs, emprunte beaucoup aux idées de R ousseau et fait une large place au gouvernement semi-direct. T out ce qui peut se rattacher à ce type de théories est aussi susceptible d'être mentionné par la doctrine comme une caracteristique essentielle du " régime d'assemblée » - il en est notamment ainsi du « mandat impératif » (P. Ardant, op. cit., p. 338).

52. P. A rdant, décrivant la « théorie » du régime d'assemblée, explique par exemple (op cit., p. 338) « le régime est monocaméral, ou tout au moins il n'y a qu'une assemblée qu compte ». Ce critère est égal ement retenu par le manuel de C. Debbasch (op. cit., p. 154), pour lequel, dans le régime d'assemblée, "le pouvoir législatif doit être remis à une seule assemblée ». Cette caractéristique était égal ement mentionnée par M. H auriou (op. cit. p. 65), mais aussi chez I. Laferriere (op. cit., p. 747 et 748). Elle fart même partie de la définition du « régime d'assemblée » fournie par J. Gicquel et $P$. A vril dans leur lexique de $D$ roit constitutionnel (op. cit., p. 106); ces derniers semblent faire du monocamérisme un critère essentiel de distinction du régime d'assemblée, puisqu'à l'entrée "Régime directorial », on peut lire que « parfois rangé dans la catégorie des régimes d'assemblée, le régime suisse s'en distingue cependant par le bicamérisme, exclusif de l'hégémonie d'une assemblée » (ibid., p. 106).

53. J. Barthélémy et P. Duez (op. cit., p. 148) indiquent par exemple que « très souvent, dans cette combinai son, (l'exécutif) prend la forme collégiale qui en accentue, encore, la fai- 
Si ces critères de détermination sont nombreux, ils ne sont bien sûr jamais utilisés tous ensemble par un même auteur : ils ne désigneraient alors aucun système politique concret et la classe des régimes d'assemblée devrait al ors être dite « vide ». Ce n'est évidemment pas ainsi que procède la doctrine. D evant une telle diversité, soit les auteurs renoncent à énumérer l'ensemble de ces caractéristiques et n'en retiennent que quelques-unes comme critères véritables du « régime d'assemblée $»^{55}$, soit (le plus souvent) ils en donnent bien une description détaillée, mais $c^{\prime}$ est pour les présenter comme des « traits normaux $»^{56}$ de ce type de régime ${ }^{57}$. Ceux-ci ne tiennent pas pour autant lieu de véritables critères,

blesse ». Cette caractéristique est particulièrement explicitée chez C. Debbasch (op. cit., p. 154) : "l'exécutif n'est pas, en règle générale, remis à un seul individu, mais à un organe collégial dans le souci d'éviter que l'exécutif n'acquière une trop grande puissance. La collégialité entraîne une diffusion des quelques " pouvoirs » remis à l'exécutif et crée naturellement une méfiance réciproque, une concurrence, qui limitent les pouvoirs de chaque membre ». Le critère de la collégialité est égal ement mentionné par $M$. Prélot et $J$. Boulouis (op. cit., p. 102).

54. Ills sont toutefois tous deux contestés, comme critères du "régime d'assemblée »; P. Bastid par exemple, s'il admet « que l'unité de Chambre est favorable au gouvernement d'assemblée », explique que "le monocamérisme ne joue (... pas un rôle absolument indispensable et il n'y a pas lieu de le retenir dans une définition » (op. cit., p. 388). De même, pour M. Prél ot et J. Boulouis, « le gouvernement d'Assemblée ne dépend pas de l'unicité de 'A ssemblée, comme organe politique, ni même du monocamérisme (en Suisse, I'A ssemblée nationale est formée des deux Conseils réunis) » (op. cit., p. 101). M. Duverger explique également: " on a tendance en France à confondre le « régime d'assemblée » et le système de la Chambre unique, qui est tout différent » (op. cit., p. 194).

55. Cette méthode, très largement utilisée par la doctrine, n'est pourtant que très rare ment présentée comme telle : même s'ils privilégient certains critères plutôt que d'autres, les auteurs préfèrent accumuler les caractéristiques du "régime d'assemblée », quitte à laisser entrevoir que certaines sont plus essentiell les que d'autres relativement à la définition de cette catégorie. J. Barthélémy et P. Duez (op. cit., p. 148 à 150) semblent toutefois s'approcher de cette présentation, puisqu'ils font de l'existence d'un chef de l'État irresponsable d'une part, et du pouvoir des chambres de nommer, diriger et révoquer le gouvernement, les critères véritablement distinctifs du "régime d'assemblée ». L'absence de droit de dissolution par exemple n'est pas considérée comme un élément de la définition du régime d'assemblée, puisqu'ils qual ifient l'existence de ce droit dans la Constitution prussienne de 1920 de « correctif » (ibid. p. 149), et que l'absence de chef de l'État dans cette constitution suffit pour eux à la faire figurer dans cette catégorie. Ce classement est toutefois nuancé, en raison de ce droit de dissolution, puisqu'ils ajoutent (ibid., p. 150), comme pour mieux souligner le caractère vague des critéres de leur notion de « gouvernement d'assemblée » : "la constitution prussienne juxtapose, dans un même texte, deux conceptions opposées: le régime parlementaire, le régime conventionnel ». Quant à la collégialité, elle n'est explicitement présentée que comme une spécificité habituelle mais non essentielle de ce type de régimes (elle n'apparaît, sel on leurs propres termes, que « très souvent, dans cette combinaison »; ; ibid., p. 148).

56. Selon l'expression de J. Laferrière, qui expliquait (op. cit., p. 748), avant d'en venir à la description des caractéristiques essentielles du " régime d'assemblée » : "Cette façon de concevoir la situation de l'Exécutif comporte, quant à son organisation, des conséquences qui, sans être des règles absol ues de ce système, en sont des traits normaux, destinés qu'ils sont à en réaliser l'idée maîtresse, c'est-à-dire la subordination de l'exécutif aux assemblées » (nous soulignons).

57. Les auteurs évoquent alors volontiers la « logique » du gouvernement d'assemblée, son " esprit » (P. Bastid, op. cit., p. 391) ou la « conception », "l'idée » qui y préside (L. Duguit, M anuel... op. cit., p. 384-385): ces expressions leur permettent d'accumuler les caractéristiques propres au "regime d'assemblée », tout en justifiant le fait que les systèmes 
dans la mesure où l'absence de l'un (ou plusieurs) d'entre eux dans un régime concret n'entraîne pas automatiquement son exclusion de la caté gorie. $\mathrm{N}$ otons cependant qu'en définitive, ces deux présentations ne se distinguent qu'en apparence : concrètement, il revient absolument au même d'user de l'une ou de l'autre ${ }^{58}$.

$D$ ans ces conditions, la grande diversité des classements proposés par les auteurs n'est pas surprenante : à retenir par exemple l'absence d'un chef de l'État comme critère essentiel du régime d'assemblée et à écarter - plus ou moins explicitement - comme tel l'inexistence du droit de dissolution (ou l'inverse), chacun en arrive à classer dans cette catégorie des systèmes politiques fort différents. Mais ces divergences doctrinales posent surtout la question de l'utilité scientifique d'une notion aussi peu consensuelle que celle-ci.

\section{2 - IN UTILITÉ SCIENTIFIQUE DE LA NOTION}

La catégorie des "régimes d'assemblée » présente bien entendu les faiblesses qui caractérisent la classification traditionnelle des régimes politiques. La démonstration a été faite que ces oppositions entre types de régimes - présidentiel, parlementaire, mais aussi, donc, "d'assemblée »-, à la « val eur logique » contestable, ne présentent en outre que peu d'intérêt scientifique ${ }^{59}$. La distinction la plus classique (entre régimes parlementaires et présidentiels) comporte toutefois un intérêt pratique évident, quoique restreint : même si toute la doctrine ne s'accorde pas sur les régimes qui méritent d'être classés dans I'une ou l'autre catégorie, et quoiqu'il s'agisse d'une opposition mal définie, l'usage de cette terminologie, permet d'obtenir rapidement quelques informations

politiques concrets classés dans cette catégorie puissent ne pas les remplir toutes - ceux-ci n'étant pas censés aller au bout de cette " logique », de cet " esprit » ou de cette « conception ». N otons qu'en tout état de cause, lorsque les auteurs accumulent ainsi les spécificités du régime d'assemblée, leur énumération n'est jamais aussi exhaustive que celle que nous avons proposée : il existe toujours une certaine sélection, de la part des auteurs, des traits censés être les plus caractéristiques du "régime d'assemblée ».

58. Une troisième forme de présentation de ces critères, consiste pour les auteurs à décrire non pas la classe des régimes d'assemblée, mais l'exempletype de cette catégorie (la Suisse essentiellement, parfois la Convention nationale), et de proceder par voie de généralisation. $C^{\prime}$ est ce que font $M$. Prélot et J. Boulouis (op. cit., p. 101 à 103) et dans une moindre mesure (parce qu'il procède aux deux types de descriptions) A. Esmein (É léments... , 8 éd., p. 533 à 538). On percoit immédiatement ce que cette presentation a de trompeuse : elle a seule ment pour conséquence de brouiller la distinction opérée nécessairement par les auteurs entre les caractéristiques essentielles du "régime d'assemblée » (qui font que l'exemple concerné a été choisi) et celles qui ne sont qu'accessoires (qui tiennent seulement aux specificités du pays concerné).

59. La démonstration en a été faite, en ce qui concerne les régimes parlementaire et pré sidentiel, par M. Troper : " Les classifications en droit constitutionnel », op. cit., p. 251 et s. La catégorie des « régimes d'assemblée » est évidemment justiciable des mêmes critiques, appuyées par les mêmes arguments. 
sur l'existence de quel ques règles juridiques simples au sein de tel ou tel régime concret ${ }^{60}$. L'emploi de ces expressions autorise, en fin de compte, une « communication rudimentaire ${ }^{61}$ sur les systèmes politiques en question.

Or, l'on peut sérieusement douter que le concept de « régime d'assemblée » réussisse même à remplir cette fonction - pourtant minimale. Les discordances doctrinales quant à la définition du « régime d'assemblée » sont en effet telles, que les auteurs qui, sans prendre parti sur le fond de la question, tentent de donner une définition seulement lexicale de ce concept, sont contraints de distinguer des sous-catégories ${ }^{62}$. La profusion et la diversité des exemples de régimes d'assemblée ainsi que des critères distinctifs de cette catégorie font qu'il est bien difficile d'indiquer des constantes au sein des définitions proposées ${ }^{63}$.

Ainsi, à la différence des régimes parlementaire ou présidentiel, on ne peut même pas se satisfaire du possible usage lexical du concept de " régime d'assemblée ». Lorsque ce terme est utilisé, on ne peut rien en déduire relativement à quel que disposition ou pratique constitutionnelle que ce soit, dans le systeme dont il est question. La seule chose en définitive que l'emploi de l'expression "régime d'assemblée » nous apprenne, concerne l'opinion de son utilisateur : ce dernier indique par cet usage que le systeme qu'il évoque fait une place à ses yeux trop importante à l'organe législatif aux dépens de l'organe exécutif.

II est dès lors légitime de se demander pourquoi les auteurs divergent de manière aussi spectaculaire, tant sur la définition de cette caté-

60. G. Burdeau, F. H amon et M. Troper expliquent à propos de ces types de régimes (op. cit., p. 112) que " lorsqu'on dit d'un pays qu'il connaît un systeme parlementaire, (...) chacun comprend qu'il existe là-bas une forme quelconque de responsabilité politique du ministère. Cette indication ne renseigne pas sur le siège réel du pouvoir, ni sur le fonctionnement effectif du système, mais seulement sur l'existence d'une règle ». De même " dire d'un régime qu'il est présidentiel, c'est dire qu'il existe un chef d'Etat élu au suffrage universel, qu'il n'est pas politiquement responsable et qu'il ne peut dissoudre les chambres. Là encore, même si I'on n'a pas ainsi communiqué d'information sur le fonctionnement effectif du système (...), on a signifié l'existence de deux ou trois règles simples ». p. 112)

61. L'expression est tirée du manuel de G. Burdeau, F. H amon et M. Troper lop. cit.,

62. C'est le cas par exemple de M. de Villiers (op. cit., p. 185) qui distingue trois cas de figure parmi les régimes d'assemblée: celui des systèmes qui résultent de l'organisation des pouvoirs instituée par les assemblées constituantes; celui des régimes qui sont le fait de choix constituants; et celui qui résulte du " dévoiement » des régimes parlementaires. C'est égal ement le cas du manuel de G. Burdeau, F. H amon et M. Troper (op. cit., p. 98) qui distingue quant à lui - mais d'une manière critique - trois exemples (plus que trois " cas de figure ») : le régime institué par la Convention nationale entre 1792 et 1795, celui qui était prévu par la Constitution française de 1793 et celui de la Confédération hel vétique. Ills ajoutent les pratiques institutionnelles des III et IV e Républiques d'une part, et les régimes socialistes, quoique avec quel que réserve, de l'autre.

63. P. Bastid lui-même reconnaissait (op. cit., p. 1) que la confusion qui entourait la notion de « régime d'assemblée » tenait à « la diversité des régimes que l'on fait communément rentrer sous cette rubrique. II est à coup sûr malaisé de formuler une définition qui puisse convenir à la fois au système de la Convention et à celui de la Confédération suisse ». 
gorie, que sur les régimes politiques qu'il convient d'y ranger. Aussi estil souhaitable de s'interroger sur l'apparition de cette notion dans le champ conceptuel des auteurs de droit constitutionnel. On peut en effet espérer que la détermination de ses origines permettra de mieux saisir la fonction remplie par la notion de "régime d'assemblée » au sein du discours doctrinal.

\section{II - L'APPARITION DE LA NOTION DE «RÉGIME D'ASSEMBLÉE » (COMME CATÉGORIE DE RÉGIMES POLITIQUES)}

L'idée de confier à l'organe législatif une place prépondérante dans le système institutionnel n'est évidemment pas nouvelle. Les plus démocrates des révolutionnaires français s'en réclamaient déjà, afin de critiquer celui que la Constitution de 1791 avait établi - et qui tendait, lui, à l'équilibre entre les organes. Les projets girondin et montagnard de 1793 se posaient d'ailleurs comme l'expression constitutionnelle de ces conceptions démocratiques, dont J ean-J acques R ousseau était le repère philosophique essentiel ${ }^{64}$. De même, l'amendement présenté par Jules G révy en 1848 s'inscrira, comme d'autres, plus tard, dans cette tradition ${ }^{65}$.

Les objections à cette conception que l'on peut dire « démocrate » ne sont bien sûr pas plus récentes : elles consistent à mettre en évidence les risques que l'adoption de ce système engendre, de voir l'organe législatif abuser des pouvoirs qui lui ont été reconnus ${ }^{66}$. Dès lors, désireux de frapper les esprits, les auteurs de ces critiques se plaisent à en appeler à l'histoire de France, pour démontrer la réalité des dangers qu'ils prédisent. Aussi invoquent-ils l'exemple de la Convention et opposent-ils souvent le souvenir de la Terreur à ceux qui s'appuient sur la « logique » démocratique à seule fin de conférer plus de poids à l'assemblée législative élue par les citoyens ${ }^{67}$. C'est pour cela, d'ailleurs, que l'on voit l'expres-

64. Cf. Michel Troper, La séparation des pouvoirs dans I'histoire constitutionnelle francaise, Paris, LGDJ, 1980, p. 142 à 154. Ce sont d'ailleurs bien ces références que donnent les auteurs lorsqu'ils prétendent décrire ce qu'ils appellent la « théorie » ou la « logique » du régime d'assemblee.

65. J ules Grévy concluait son discours, le vendredi 6 octobre 1848 en indiquant, de manierre significative (Le M oniteur U niversed, Journal officid de la République française, p. 2735): «Dans un gouvernement populaire, le pouvoir est dans une assemblée; c'est là sa condition, comme la condition du pouvoir dans une monarchie est d'appartenir à un homme. (...)' Le pouvoir sera dans une A ssemblée. D ans une démocratie, il ne peut, il ne doit pas être ailleurs ».

66. A cet égard, ces critiques ressemblent fort à celles qui seront adressées, plus tard, à la catégorie des « régimes d'assemblée » par la doctrine constitutionnelle.

67 . Ainsi Odilon Barrot, sous la Constituante de 1848 , critiquait-il, un peu abusivement, le monocamérisme défendu par Lamartine (séance du 27 septembre 1848, L e M oniteur U niverse, J ournal officiel de la République française, p. 2622) : "Oui, il s'agit de savoir si, par 
sion de « système conventionnel » apparaître bien avant celle de " régime d'assemblée ${ }^{68}$ - qui finira pourtant par lui succéder. Mais elle ne désigne al ors pas une catégorie abstraite de régimes politiques - pour la bonne raison que l'idée même de classer les régimes sel on des catégories abstraites n'a pas encore vu le jour -, mais seulement un système s'apparentant à celui institué concrètement par la Convention en 1792.

C'est cependant bien à l'émergence de la notion de " régime d'assemblée » comme catégorie abstraite, s'inscrivant dans la classification traditionnelle des régimes politiques, que l'on a choisi de s'intéresser ici - quand bien même celleci aurait des racines théoriques et pratiques plus lointaines. Or, pour découvrir les origines de cette notion, il convient de remonter très loin dans le temps : aussi loin, en réalité, que la classification des régimes politiques elle-même. Aussi est-il nécessaire d'examiner les circonstances dans lesquelles cette classification a vu le jour (1), pour être en mesure d'analyser l'émergence de la notion de "régime d'assemblée » (2).

votre constitution, vous allez organiser une dictature... Une dictature collective, je le reconnais, mais une dictature, un pouvoir unique, tout-puissant, sans contre-poids, sans responsabilité, car les responsabilités collectives, I'histoire nous apprend ce qu'elles sont. (...) Lorsque nos pères, dont on cite toujours l'exemple et qu'on imite à faux presque toujours, lorsque nos pères faisaient un gouvernement révolutionnaire, ils avaient la franchise de le dire, ils le décrétaient sous l'influence de la nécessité du moment; mais ils ne faisaient pas une constitution pour organiser d'une manière permanente un gouvernement révolutionnaire, une convention permanente. En avez-vous un seul exemple dans le monde entier ? (...) C'est donc la Convention que vous allez décréter, avec sa toute-puissance, n'ayant à côté d'elle aucun pouvoir modérateur, un pouvoir exécutif qui s'absorbe en elle».

D'une manière très similaire, sous l'A ssemblée nationale constituante élue en 1871 Édouard Laboulaye plaidera pour une seconde chambre en se référant également à la Convention (séance du 28 février 1873, A nnales de l'A ssemblée nationale - Compte rendu in extenso des séences, t. XVI, p. 240) : "je maintiens qu'une seconde chambre est un élément nécessaire pour une démocratie qui veut durer. Q ue cette seconde chambre doive être nommée, comme la première, par le suffrage universel, c'est mon opinion. Mais prétendre que I'on peut gouverner un pays, et un grand pays, avec une A ssemblée de sept cents membres, c'est retomber dans les doctrines de la Convention, c'est vouloir en arriver au régime des comités, au régime du salut public, et finir par le césarisme ». Cette utilisation de la Çonvention nationale comme épouvantail ne concernait du reste pas que le monocamérisme. Édouard Laboulaye en avait déjà fait usage bien avant d'être élu a l'A ssemblée nationale. En 1851, il écrivait en effet : " Un président doit, en certains cas, arrêter ou empêcher la décision de l'A ssemblée, sinon il n'est rien, et il est plus simple d'en revenir au gouvernement des Comités. La tyrannie sera plus apparente et le pays s'y trompera moins longtemps » (L a révision de la Constitution - L ettres à un ami, Paris, A. Durand, 1851, p. 126). Et en 1871, il usait à nouveau de cette rhétorique à propos de la nécessai re indépendance de l'organe exé cutif : " Faire du président le simple ministre, le serviteur de l'assemblée, c'est fonder le despotisme du Corps législatif » (La République constitutionnelle, Paris, Charpentier et Cie, 1871 , p. 16).

68. Dès 1848, lors des débats constituants, Jules de Lasteyrie évoquait ainsi, parmi les systèmes constitutionnels proposés, celui contenu dans l'amendement Grévy : " on nous propose le systeme conventionne, c'est-àdire la confusion du pouvoir exécutif avec le pouvoir législatif, et cela, messieurs, pour appliquer dans un temps ordinaire, dans des circonstances normales, ce que la convention ellemême n'a cru pouvoir appliquer que pendant deux années » (séance du 6 octobre 1848, Le M oniteur U niverse, J ournal officied de la République française, p. 2735 ; nous soulignons). 


\section{1 - AUX ORIGINESDE LA CLASSIFICATION THÉORIQUE DES RÉGIMES POLITIQUES}

Beaucoup de choses ont été écrites sur cette classification. Toujours très utilisée par les auteurs, elle a été critiquée, mais aussi défendue sous de multiples points de vue. Chacun des régimes qu'elle s'efforce de distinguer a fait l'objet de nombreuses analyses; on en a examiné les définitions, les caractéristiques, ainsi, bien sûr, que les origines théoriques et pratiques. En revanche, il semble que l'histoire de cette classification en tant que telle n'ait pas été faite. Quelques articles ou passages évoquent la question ${ }^{69}$, mais certaines des circonstances ayant entouré l'émergence de cette idée restent bien obscures.

II semble que la doctrine n'ait pas songé à opposer les régimes parlementaire et présidentiel avant la fin du XIXe siècle. Avant cela, elle se contentait de rendre compte du fonctionnement des systèmes politiques concrets soumis à son examen - non, d'ailleurs, sans quelque effort de systématisation. Richard Moulin explique ainsi parfaitement comment, au cours du XIXe siècle, les auteurs ont su observer combien les pratiques institutionnelles américaine et britannique, pourtant initialement fondées sur des systèmes constitutionnels très proches, s'étaient progressivement éloignées l'une de l'autre ${ }^{70}$. En Angleterre, puis, un peu plus tard, en France, la balance des pouvoirs va évoluer vers un système dans lequel le veto législa tif disparaît et où le Parlement obtient progressivement le droit de contrôler (dans tous les sens du terme) le cabinet ministériel. Aux États-U nis, au contraire, non seulement le Président conserve son droit de veto, mais il continue de nommer ses ministres de manière absolument discrétionnaire, qui ne forment pas, comme en Europe, un cabinet ministériel responsable devant les chambres. Ainsi que le note Richard M oulin, « il était al ors aisé d'établir un antagonisme entre les deux types de pratiques ${ }^{71}$, ce que, pré cisément, ne manquent pas de faire bon nombre d'auteurs ${ }^{72}$. Pour opposer l'expérience américaine au système européen (que l'on qualifiait al ors déjà de « parlementaire »), la doctrine parle de plus en plus, à la suite de W alter Bagehot en Angleterre, de gouvernement « présidentiel $»^{73}$.

69. Par exemple Richard M oulin (op. cit.), H ugues Tay (Le régime présidentid et la France, Paris, LGDJ, 1967, p. 3 et S.) ou Gérard Conac ("Le régime presidentiel », in D ictionnaire constitutionne, 0 . Duhamel et $Y$. Mény (sous la dir.), Paris, PUF, 1992, p. 896).

70. R. Moulin, op. cit., p. 10-11.

71. Ibid., p. 11.

72. R. M' oulin indique que l'on « opposait ainsi le régime représentatif des États-U nis au régime parlementaire de la G rande-Bretagne » (ibid., p. 11).

73. Cependant, ces expressions de « régime parlementaire » ou " présidentiel » ne désignent alors qu'une pratique concrète du pouvoir et ne sont pas encore, au moment où elles apparaissent, opposées comme des "classes » de régimes politiques. Aussi convient-il de prendre garde a ne pas confondre l'apparition des mots avec celle des concepts : les expressions « régime présidentiel » et " régime parlementaire » voient le jour à des moments différents, avant que les concepts que l'on désigne aujourd'hui sous ces vocables n'émergent, conjointement, que lorsque apparaît la classification des régimes politiques. 
Cependant, il semble que la doctrine française ne se soit pas contentée de rendre compte des divergences existant entre les pratiques institutionnelles américaine et européenne, et se soit par conséquent efforcée de « les systématiser, pour dépasser la simple description, et aboutir à la création de classes $\gg^{74}$. Ce sont justement les raisons pour lesquelles les auteurs français ont subitement choisi d'établir une telle classification (que l'on dit parfois abstraite), au lieu de continuer à décrire des régimes concrets, qui restent bien mystérieuses. Selon une idée très répandue, cette orientation - impulsée notamment par Adhémar Esmein ${ }^{75}$ - serait à mettre sur le compte de l'esprit de système propre à la doctrine française de l'époque, de son " goût des belles ordonnances classiques ${ }^{76}$. Cette explication, d'ordre psychologique ou culturel, n'est cependant guère convaincante. Dès lors, il peut être utile de pousser plus loin la réflexion et d'examiner le contexte dans lequel la classification traditionnelle des régimes politiques, fondée sur la séparation des pouvoirs, est apparue.

Aussi convient-il d'abord de remarquer que le constitutionnaliste anglais W alter Bagehot ne s'était pas contenté, dans son œuvre majeure, d'introduire l'expression de régime " présidentiel » (presidential government ou presidential system) ${ }^{77}$ pour qualifier le système politique américain. Son propos consistait surtout à opposer ce dernier au système anglais - qu'il nomme le gouvernement « de cabinet » (cabinet government) ${ }^{78}$. L'intention de Bagehot n'est certes pas d'établir des classes de régimes sous lesquelles pourraient se ranger l'ensemble des systèmes politiques existants. N éanmoins, il s'attache à distinguer les deux pratiques institutionnelles sur la base d'un critère tiré du principe de la séparation des pouvoirs. Sa distinction n'est toutefois pas celle que l'on connaît aujourd'hui : pour lui, le gouvernement présidentiel est un régime de séparation des pouvoirs, tandis que le gouvernement de cabinet est au contraire un système de confusion des pouvoirs au profit du Parlement ${ }^{79}$. Sel on ses propres termes, " le secret véritable de la Constitution anglaise peut être décrit comme l'union intime, la fusion presque complète des pouvoirs législatif et exécutif. Le trait d'union est le Cabinet. Par ce mot

74. R. Moulin, ibid., p. 12.

75. Celui-ci est en effet le plus souvent présenté comme l'inventeur principal de cette classification théorique des régimes politiques. C'est bien ainsi que Gerard Conac (" Le régime présidentiel », op. cit., p. 896) ou H ugues Tay (op. cit., p. 8) le présentent.

76. G. Conac, "Le régime présidentiel », op. cit., p. 896.

77. W. Bagehot, The Engl ish C onstitution, in "The collected W orks of W alter Bagehot », vol. V, "The political essays », ed. by N. St John-Stevas, London, The Economist, 1974, p. 214.

78. I bid.

79. II explique que "I'indépendance des pouvoirs législatif et exécutif est la qualité spé cifique du systeme présidentiel, de même que leur fusion et leur combinai son est le principe typique du gouvernement de cabinet » (W. Bagehot : ibid., p. 214 ; nous traduisons). 
nouveau nous désignons un Comité du Corps législatif élu pour être le Corps exécutif $»^{80}$.

Or, cette thèse ${ }^{81}$ commence, dans la France de la fin du XIxe siècle, à rencontrer un certain succès ${ }^{82}$. D'un point de vue institutionnel d'abord, le système français semble, à la suite de la célèbre crise du 16 mai 1877, se rapprocher du tableau dressé par Walter Bagehot ${ }^{83}$. Le parlementarisme à l'européenne se distingue alors par plusieurs traits caractéristiques: le chef de l'État s'effaçant toujours plus, le gouvernement est, dans les faits, choisi directement par le parlement parmi ses propres membres; le ministère est en outre conçu comme devant mettre en œuvre la vol onté de la représentation nationale, incarnée dans les seules chambres; ces dernières peuvent du reste, en cas de désaccord politique avec le gouvernement, le démettre de ses fonctions. Dès lors, on peut penser que « l'action exécutive elle-même apparaît, en définitive, comme dépendant, par l'intermédiaire des ministres, de la volonté parlementaire. Dans ces conditions, le ministère doit être considéré comme étant essentiellement une commission gouvernementale des assemblées ${ }^{84}$.

80. Cité par A. Esmein, Éléments... , 1896, note 1, p. 312. Voici la version originale de cet extrait : "The efficient secret of the English Constitution may be described as the close union, the nearly complete fusion, of the executive and legislative powers. (...) The connecting link is the Cabinet. By that new word we mean a committee of the legislative body selected to be the executive body » (W. Bagehot, The English Constitution, op. cit., p. 210).

81 . Telle du moins qu'elle a été comprise par certains auteurs francais. Certains passages de l'œuvre de W. Bagehot semblent en effet moins clairs, de ce point de vue, que ceux habituellement cités en France. II écrit par exemple, de manière un peu obscure: " The English system (...) is not an absorption of the executive power by the legislative power ; it is a fusion of the two » (W. Bagehot, ibid., p. 213).

82. De fait, la traduction de l'ouvrage de W alter Bagehot parait en France en 1869 (La Constitution anglaise, trad. fr. M. Gaul hiac, Paris, Germer Barillière, 1869). Et selon Adhé mar Esmein, la thèse (qu'il attribue à Bagehot) sel on laquelle le régime parlementaire est un système de confusion des pouvoirs constitue, au moment où il écrit (1896), une idée « assez répandue en France » (A. Esmein, Eléments de droit constitutionne, 1896, p. 312). Raymond Carré de Malberg fera, plus tard, la même observation (C ontribution à la thérie génerale de I'E tat, Paris, Recueil Sirey, t. II, 1922, p. 71-72).

83. C'est d'ailleurs à partir des transformations radicales qui affectent al ors le régime parlementaire français, autour des années 1880-1890, que, comme le fait remarquer $\mathrm{H}$. Tay, « un certain nombre d'auteurs (paraissent) soucieux d'approfondir la distinction des régimes politiques sel on les données de la technique constitutionnelle, et, surtout, sel on les rapports des organes gouvernementaux » $(H$. Tay, op. cit., p. 7). Toutefois, comme il le démontre ensuite, aucun de ces auteurs n'oppose encore radical ement des « types » de régimes selon la faccon dont ils mettent en œuvre la séparation des pouvoirs - comme le fera plus tard Esmein.

84. C'est ainsi que R. Carré de Malberg (C ontribution... , op. cit., t. II, p. 72) rend compte de cette opinion doctrinale (sans pour autant y adhérer sur le fond). II résume plus loin cette interprétation du régime parlementaire avec la concision qui le caractérise: "En somme, la conclusion qui se dégage de cette première doctrine, c'est que les Chambres sont l'autorite initiale et suprême, aussi bien en ce qui concerne la puissance exécutive qu'en matière de législation. De ces deux puissances, elles exercent l'une par elles-mêmes, l'autre par un Cabinet qui procède d'elles seules et qui ne dépend aussi que d'elles. Sel on cette conception, le pouvoir exécutif ne forme plus, par sa constitution organique, un second pouvoir princi$\mathrm{pal}$ et essentiellement distinct. M ais la vérité est que les Chambres réal isent en elles I'unité de la puissance d'Etat. C'est en ce sens surtout que le régime parlementaire apparaît al ors 
D'un point de vue politique, ensuite, le contexte de forte hostilité au Parlement et aux parlementaires ${ }^{85}$, qui traverse alors la classe politique française, favorise évidemment la popularité de la thèse de Bagehot. La critique se concentre à l'époque sur le pouvoir des chambres, et l'on est dès lors enclin à dénoncer leur omnipotence. Présenter le régime parlementaire, qui est celui de la IIle République, comme un régime de concentration des pouvoirs au profit du Parlement permet seulement de porter la critique sur un plan strictement institutionnel.

Mais c'est précisément cette interprétation du régime parlementaire que certains auteurs, et en particulier Esmein, ne peuvent accepter ${ }^{86}$. II est essentiel, pour ces auteurs, de montrer que ce système respecte en principe la règle de la séparation des pouvoirs, car il s'agit, de leur point de vue, d'une garantie aussi traditionnelle qu'essentielle de la libertés7. Ils ne peuvent par conséquent admettre que les institutions de la III R épublique soient comprises comme instituant, par nature, le despotisme d'un organe - quoique ces auteurs aient pensé, par ailleurs, de la manière dont ces institutions étaient pratiquées.

C'est bien du reste A dhémar Esmein qui s'attache à contrecarrer cette interprétation, essentiellement dans ses Éléments de droit constitutionnel, parus en 1896. II le fait de façon très efficace, mais aussi fort simple. Comme Walter Bagehot, il oppose le système américain aux systèmes anglais et européen, mais il s'efforce, on l'aura compris, de proposer un autre critère de distinction. L'idée, fort ingénieuse, d'Esmein, consiste précisément à intégrer l'idée que le régime parlementai re est respectueux du principe de séparation des pouvoirs dans le critère de distinction luimême. Ainsi, au lieu de substituer à celui avancé par Bagehot un critère fondé sur de tout autres considérations, Esmein propose justement de concevoir les régimes anglais et américains comme deux applications différentes de la régle de la séparation des pouvoirs. II prétend que, dans le

comme excluant l'idée de séparation des pouvoirs » (ibid. p. 74). II ajoutait immédiatement à ce propos: "Mais c'est aussi et précisément contre cette conclusion que s'élève un second groupe d'auteurs, au premier rang desquels se place M. Esmein » (ibid.).

85. Contexte dont Marie Joëlle Redor a longuement rendu compte (D e l'État légal à l'É tat de droit. L'évolution des conceptions de la doctrine publiciste française : 1879-1914, Paris, "D roit public positif », PUAM-Économica, 1993, p. 88 et s.)

86. A. Esmein proteste ainsi énergiquement (Éléments.... 1896, p. 312) : " On a, à cet égard, donné des formules bien exagérées et qui tendent à devenir courantes. On dit que le gouvernement parlementaire n'est pas autre chose que le pouvoir exécutif exercé par un Comité que nomme la Chambre populaire et qu'elle peut révoquer à son gré. Cela a été dit pour l'Angleterre (...) par M. Bagehot (...) ; c'est une idée assez répandue en France. M ais ce n'est pas là le droit (...)».

87. G. Conac explique parfaitement («Régime présidentiel», in D ictionnaire constitutionne, dir. O. Duhamel et $Y$. M ény, op. cit., p. 896) que « si les auteurs français classiques attachent autant d'importance à la séparation des pouvoirs législatif et exécutif, c'est pour une raison idéologique. Pour eux, elle est liée à l'essence même du constitutionnal isme libéral. C'est pourquoi par exemple pour A. Esmein, il est important de soutenir qu'elle subsiste dans le régime parlementaire anglais qu'il admire. Aussi critiquet-il les conclusions de W alter Bagehot qu'il juge excessives ». 
système américain, le principe y est appliqué strictement, tandis qu'il ne l'est que de manière « atténuée » en Angleterre ${ }^{88}$. D ès lors - en vertu de l'ontologie réaliste qui caractérise le raisonnement juridique ${ }^{89}-$, le système " parlementaire » anglais, mais aussi français, apparaît comme étant par nature respectueux du principe de la séparation des pouvoirs.

Le critère tiré de ce principe est donc bien, comme le soulignent traditionnellement les auteurs, éminemment abstrait. Mais son choix n'a aucunement résulté de l'esprit systématique de la doctrine française de l'époque, mais seulement de la nécessité dans laquelle une partie d'entre elle s'est trouvée, de répondre à une thèse qu'elle jugeait dangereuse.

\section{2 - L'ÉMERGENCE DE LA NOTION DE «RÉGIME D'ASSEMBLÉE »}

À ce point, la démonstration d'Esmein est toutefois encore incomplète. S'il s'était contenté d'opposer ainsi deux façons d'appliquer le principe de la séparation des pouvoirs, il se serait probablement heurté à une objection de taille. Si le régime parlementaire se définit, par opposition à la séparation stricte des États-U nis, comme un système qui comporte des exceptions à la règle de la séparation des pouvoirs, qu'estce qui distingue réellement cette séparation dite "atténuée » ou " souple», de la confusion des pouvoirs? En d'autres termes, est-ce que la souplesse, en tant qu'elle autorise la transgression du principe, n'équivaut pas à la confusion ? Esmein anticipe en fait cette objection en usant du même procédé que précédemment.

Son ambition, il l'indique lui-même, consiste à montrer que le régime parlementaire, certes, " atténue la séparation des pouvoirs, mais la maintient cependant $»^{90}$. II s'attache par conséquent à mettre en évidence que l'éventuelle domination politique des chambres sur le gouvernement n'entraîne pas irrémédiablement la confusion des pouvoirs. Sel on lui, ce danger est notamment évité, en régime parlementaire, par l'existence d'un certain nombre d'institutions et de règles spécifiques, qui limitent le pouvoir de l'organe législatif. En d'autres termes, la confusion des pouvoirs au profit de l'assemblée ne se réal iserait que si ces règles et ces institutions spécifiques étaient absentes.

88. Cette idée selon laquelle le principe de la séparation des pouvoirs pourrait être plus ou moins strictement appliqué n'est pas tout à fait nouvelle. Antoine de Saint-Girons s'en fai sait déjà l'écho en 1881 : Il y suggérait qu'entre « la confusion et la séparation, se trouve un moyen terme qui n'a aucun des inconvénients signalés par I'histoire : c'est l'union des pouvoirs appliquée en France de 1814 à 1848 » (A. de Saint-Girons, E ssai sur la séparation des pouvoirs dans l'ordre politique, administratif et judiciaire, Paris, Larose, 1881, p. 291). En revanche, c'est bien Esmein qui élève cette distinction en critère de l'opposition entre régimes " parlementaires » et " présidentiels ».

89. Qu'il soit permis à ce sujet de renvoyer à la démonstration déjà mentionnée de $M$ ichel Troper, "Les classifications en droit constitutionnel », op. cit., p. 259-260.

90. A. Esmein, Éléments... , 1896, p. 311 (nous soulignons). 
Esmein n'a donc plus qu'à ajouter à son système un troisième type de régimes, distinct des régimes parlementaire et présidentiel, et dans lequel, précisément, ces règles et ces institutions spécifiques n'existent pas. C'est ce qui apparaît dans le plan de son ouvrage : après avoir évoqué la séparation stricte des États-U nis et la séparation « atténuée » des systèmes parlementaires, il traite d'une troisième catégorie de systèmes (dont il trouve pourtant bien peu d'exemples probants), qui organisent, quant à eux, la confusion des pouvoirs. Bien que l'auteur ne le qualifie pas encore ainsi, on comprend qu'il s'agit bien là du régime que l'on appellera d'abord « conventionnel $»^{91}$, puis « d'assemblée ».

Au moment où elle apparaît, la classification des régimes politiques comprend donc non pas deux mais trois catégories, et le « régime d'assemblée » y remplit une fonction fondamentale. II est destiné à justifier le régime parlementaire et à en souligner le caractère « libéral ». Cette justification opère en fait doublement.

D'une part, le concept de "régime d'assemblée » ainsi spécifié permet de présenter le régime parlementaire comme étant par définition un régime de séparation des pouvoirs - puisque c'est précisément ce qui différencie celui-ci de celui-là. C'est ce qui apparaît nettement à la lecture de la présentation qu'en donne Esmein : " Si le gouvernement parlementaire ne confond pas les deux pouvoirs, écrit-il, il est une autre forme de gouvernement, qui se propose ce but, en privant le pouvoir exécutif de toute indépendance, en le subordonnant expressément au pouvoir législatif ${ }^{\$ 2}$. A insi, la seule existence du concept antithétique de " régime d'assemblée » implique - toujours du fait de l'ontologie réaliste qui caractérise les classifications doctrinal e ${ }^{93}$ - le caractère naturellement libéral du régime parlementaire. C'est là, du reste, le tour de force réussi par Esmein : seul désormais un « dévoiement » du régime parlementaire, et non son fonctionnement normal, pourra être accusé de concentrer les pouvoirs dans les mains du Parlement.

D'autre part, en tant que le « régime d'assemblée » est défini comme un régime de confusion des pouvoirs, les règles et les institutions qui le caractérisent apparaissent comme bien peu souhaitables. À l'inverse, les traits qui distinguent le régime parlementaire de ce dernier semblent, presque par hypothèse, devoir être maintenus - car ils constituent la garantie du respect de la séparation des pouvoirs. C'est du reste à ce sujet que prennent leur source les premières divergences doctrinales relative-

91. R. Carré de Mal berg résume par exemple ainsi I'opinion d'Esmein (C ontribution... , op. cit., p. 74): «Si le gouvernement parlementaire engendrait une telle confusion (des pouvoirs), il ne serait en réal ité, qu'une forme de ce que l'on appelle, en France, depuis 1793, le gouvernement conventionnel ». L. Duguit et M. H auriou, notamment, utiliseront cette même expression.

92. A. Esmein, Éléments... , 1896, p. 314.

93. Cf. supra. 
ment à la définition du " régime d'assemblée ». En effet, les auteurs sont évidemment en désaccord sur les garanties en question : si ériger une disposition constitutionnelle en critère de distinction entre ces deux types de régimes revient à démontrer qu'elle doit subsister, l'identification desdits critères devient un enjeu idéologique et doctrinal essentiel. II n'est dès lors pas étonnant de voir les auteurs se déterminer à cet égard sel on leurs options théoriques ou leurs opinions respectives. Si Adhémar Esmein insiste par exemple particulièrement, pour caractériser le " régime d'assemblée », sur l'absence d'un chef de l'État irresponsable ${ }^{94}$, Léon Duguit souligne plutôt l'inexistence de moyens d'action réciproques entre l'Exécutif et le Législatif95, tandis que pour Maurice $\mathrm{H}$ auriou, le régime d'assemblée se résume pour ainsi dire au monocamé risme ${ }^{96}$. Chacun d'entre eux justifie ainsi l'existence des règles ou institutions correspondantes (présidence de la République, droit de dissolution, bicamérisme) dans le régime parlementaire - et donc dans celui de la IIle République.

Ces désaccords doctrinaux perdureront tant que le concept de « régime d'assemblée » sera revêtu d'une telle charge idéologique. Ils s'expliquent par la fonction que cel ui-ci a longtemps remplie dans le discours doctrinal.

\section{III - LA FONCTION DE LA NOTION DE «RÉGIME D’ASSEMBLÉE »}

La notion de régime d'assemblée a longtemps été, on l'aura compris, une sorte de « concept-repoussoir », de "spectre », pour reprendre une expression que Michel Troper a utilisée à propos de la notion de " gouvernement des juges ${ }^{97}$. Le concept de " régime d'assemblée » en pré sente en effet tous les traits caractéristiques.

II est en premier lieu destiné à effrayer, essentiellement parce qu'il est supposé confondre les pouvoirs entre les mains de l'assemblée législative ${ }^{98}$. Mais même lorsqu'il n'est pas explicitement présenté comme

94. A. Esmein, Éléments... , 1896, p. 311.

95. Pour Léon D uquit, en régime parlementaire, « la garantie de la liberté résultera pré cisément de cette action réciproque des deux organes I'un sur l'autre » (L. Duguit, M anuel... , op. at., p. 384).

96. M. 'H auriou, Précis élémentaire.., op. cit., p. 65 à 66.

97. M. Troper, "Le bon usage des spectres - Du gouvernement des juges au gouverne ment par les juges », in La theorie du droit, le droit, P'Etat, Paris, PUF, coll. "Léviathan », 2001, p. 231

98. L'absence de séparation des pouvoirs correspondant depuis le XVIII siècle à la définition du despotisme, aucun constitutionnaliste ne pourra se réclamer de cette opinion. On ne peut à cet égard que contester la neutralité dont $P$. Bastid se prévalait relativement au concept de régime d'assemblée, lorsqu'il le définissait (entre autres) comme « contraire, non seulement à toute séparation mais même à toute distinction des pouvoirs » ( $P$. Bastid, op. 
tel, le « régime d'assemblée » reste en tout état de cause connoté péjorativement. II suffit d'observer que, même compris comme un régime de séparation des pouvoirs, il est en général rangé parmi ceux organisant (par opposition aux régimes d'équilibre) la prééminence d'un organe (le législatif), aux côtés de systèmes prévoyant la préminence de l'organe exécutif - en général peu recommandables du point de vue des thèses libé ral es $^{99}$. D'ailleurs, le simple fait (communément admis) que l'on présente comme une réal isation archétypale du « régime d'assemblée » le système institué par la Convention nationale entre 1792 et 1795, atteste du caractère « inquiétant » dont la doctrine entend en général I'affecter ${ }^{100}$. Au minimum ce type de régimes apparaît-il, dans le discours doctrinal, comme peu souhaitable $e^{101}$ : il n'est jamais présenté avantageusement, comme peuvent l'être les autres types de régimes.

Le " régime d'assemblée » est en second lieu insaisissable, puisque ses réalisations concrètes sont rares ${ }^{102}$ et que les exemples avancés ne

cit., p. 13). La profession de foi qui suit immédiatement cette définition et sel on laquelle " cette affirmation n'implique aucun jugement de valeur sur la modalité politique dont il s'agit » (ibid.) est en effet bien peu credible, au regard de la tradition libérale française.

99. J. Cadart utilise cette opposition entre les « régimes de confusion des pouvoirs au profit du Parlement », dans laquelle figurent les seuls "régimes d'assemblée ", et les " régimes de confusion des pouvoirs au profit de l'organe gouvernemental » (op. cit., t. I, p. 368 à 414). Dans cette dernière catégorie, on trouve, pêle-mêle, des systèmes aussi peu " recommandables » que la monarchie absolue, les régimes théocratiques ou les " dictatures » modernes (fasciste, franquiste, nazie, communiste). On trouve d'ailleurs, dans le manuel de G. Burdeau, F. H amon, M. Troper (op, cit., p. 99-100) un compterendu des régimes généralement classés par la doctrine dans cette catégorie dite de la " prééminence du gouvernement »: "Ainsi, on y trouve selon les auteurs, différents régimes fascistes, les dictatures militaires, les régimes de parti unique les monarchies traditionnelles ou simple ment des systèmes, comme celui de la Ve République francaise, dans lesquels on constate que le Président est la figure centrale ». M is à part le dernier exemple, cette liste est édifiante, quant à la connotation qui est attachée à ce type de régimes. On peut d'ailleurs supposer que le classement de la $V^{\text {e }}$ République dans une telle catégorie - et surtout en telle compagnie ! - participe d'une critique à son égard.

100. G eorges Burdeau avait parfaitement relevé (T raité de science politique, t. IV : « Les régimes politiques », Paris, LGDJ, 1952, p. 319) la connotation attachée à cet exemple : «Quant à prendre pour prototype du gouvernement d'assemblée le régime qui fonctionna en fait sous la Convention c'est une aberration qui ne s'explique que par une intention polémique : discréditer la puissance des assemblées en leur imputant la responsabilité de la Terreur ». M. Duverger ne disait pas autre chose, lorsqu'il reconnaissait que «le terme de " régime d'assemblée » (avait) un caractère péjoratif chez nous, où il est assimilé à la Terreur, au Tribunal révolutionnaire, à la guillotine, etc. » (op. cit., t. I, p. 194).

101. P. Ardant offre un excellent aperçu de cette appréciation négative « minimale ». II distingue en effet deux significations du " régime d'assemblée » : si au sens le plus strict, ce régime organise bien une véritable confusion des pouvoirs au profit de l'assemblée, ce n'est sel on lui pas le cas dans son sens le plus large, où le parlement, suite à une "dévia tion » du régime parlementaire, se contente d'exercer une " domination forte sur l'exécutif ». II ajoute al ors, résumant bien la pensée doctrinale majoritaire à cet égard : " On ne vit plus al ors dans le drame ni dans la dictature, mais dans l'impuissance et l'immobilisme» (P. Ardant, op. cit., p. 340).

102. Benoît J eanneau écrit par exemple (D roit constitutionnel et institutions politiques, Paris, "M émentos », Dalloz, 7e éd. 1987, p. 134) : " II n'est pas contestable que le régime d'assemblée au sens strict devient une curiosité constitutionnelle et que son importance est des plus réduites dans le monde contemporain ». 
convainquent général ement pas même ceux qui les utilisent. Les auteurs s'empressent en effet souvent de nuancer le classement qu'ils viennent d'opérer, notamment lorsqu'il s'agit d'exemples étrangers ${ }^{103}$. La Suisse en particulier, qui est pourtant souvent citée comme modèle, voit son caractère exemplaire être systématiquement tempéré104 - avec seulement plus ou moins de vigueur. En ce qui concerne la France, les exemples pris ont la particularité de n'avoir jamais été complètement réalisés. II s'agit en effet soit de régimes exceptionnels simplement transitoires ${ }^{105}$ (ceux institués par les assemblées constituantes pendant leur mandat), soit de projets ou d'amendements constitutionnels repoussés ou restés inappliqués (les projets girondin et montagnard de 1793, I'amendement Grévy de 1848, le projet du 19 avril 1946), soit de pratiques institutionnelles jugées déviantes par rapport au texte constitutionnel initial (les pratiques tardives des III et IV e Républiques).

Or, c'est précisément son caractère "spectral » qui permet à ce concept de remplir les fonctions justificatives que la doctrine lui assigne. Ces dernières se situent d'ailleurs, comme à l'origine, sur un double plan. Le concept de « régime d'assemblée » permet en effet aux auteurs : d'une part, de porter une appréciation globale sur un certain nombre de régimes; d'autre part, de justifier (ou de critiquer) des règles ou des institutions très précises.

Quant au premier point, I'utilisation la plus élémentaire du concept de « régime d'assemblée » consiste à jouer de sa connotation très péjorative pour porter, par le simple classement de tel ou tel système politique concret dans ou hors de cette classe, un jugement de val eur sur ledit système ${ }^{106}$. Le seul fait de ranger dans cette catégorie tel régime concret

103. M. H auriou par exemple, cite quelques exemples étrangers où le système conventionnel est appliqué, mais il précise immédiatement qu'il est al ors toujours « plus ou moins mélangé de parlementarisme, et dans des pays de faible étendue » ( $M$. H auriou, Précis élé mentaire.. , op. cit., p. 66).

104. Ainsi, A. Esmein notait-il déjà en 1896 (Éléments... , 1896, p. 318) que, si la Suisse organise bien un tel régime - avec quelque succès - " cette harmonie particulière a été le résultat des mœurs; elle s'est établie par tradition et se maintient par esprit de sagesse ». Lorsque les auteurs n'attribuent pas, comme le fait Esmein, la réussite (ou du moins la « normalité ») du régime suisse aux particularités locales (ainsi L. Duguit, M anue..., op. cit., p. 386, M. Prélot et J. Boulouis, op. cit., p. 103, etc.) - ce qui revient à nuancer le caractère exemplaire de ce régime -, c'est son classement dans cette catégorie lui-même dont la pertinence est remise en cause (ainsi P. Ardant, op. cit., p. 343 ; J. Barthélémy et P. Duez, op. cit., p. 149 ; P. Pactet, op. cit., p. 154, etc.).

105. J. Barthélémy et $P$. Duez font par exemple remarquer (op. at., p. 149) que « le gouvernement d'assemblée n'a jamais été en France qu'un expédient exceptionnel des périodes transitoires » (nous soulignons).

106. Cela est en réalité surtout vrai en ce qui concerne les exemples français; les cas étrangers de « régimes d'assemblée », et notamment la Suisse, ne font pas nécessairement l'objet de cette éval uation : cette dernière est surtout citée pour justifier l'existence des régimes d'assemblée en tant que « catégorie » constitutionnelle - et donc pour masquer sa fonction strictement idéologique. Si en effet ce « type de régimes » n'existait qu'en France, serait-il utile d'en faire une " catégorie » à part, au même titre que les régimes parlementaire et présidentiel ? L'exemple suisse renforce en outre l'apparente symétrie que les auteurs tentent de 
revient en effet à l'accuser sinon de confondre les pouvoirs au profit de l'organe législatif, du moins d'attribuer à ce dernier des compétences démesurées. Bref, le "régime d'assemblée » fait office de " contremodèle $»^{107}$. La fonction justificative que remplit ce classement ${ }^{108}$ opère d'ailleurs dans les deux sens. Lorsque l'on se refuse à qualifier les systèmes de la III ou de la IV e République de "régimes d'assemblée »109, on atténue par là même les critiques qui ont pu être adressées à ces derniers. Mais à l'inverse, le fait de prétendre que tous les régimes parlementaires monistes peuvent être rangés dans cette catégori $e^{110}$ revient en fin de compte à critiquer le parlementarisme en tant que tel - et non plus seulement tel ou tel système politique concret ${ }^{111}$. Un tel jugement négatif sur le régime parlementaire (en tant que tel) peut d'ailleurs également résulter du classement des seules pratiques des III et IV R Républiques dans la catégorie des « régimes d'assemblée », s'il est suggéré par ailleurs que ce sont précisément les spécificités parlementaires de ces deux régimes qui ont entraîné lesdites pratiques ${ }^{112}$.

mettre en évidence avec ces deux dernières classes, puisque chacune d'entre elles dispose également de son " modèle-type » à l'étranger. Toutefois, Il arrive que certains systèmes étrangers soient stigmatisés par leur simple classement dans cette catégorie: B. J eanneau explique par exemple (op. cit., p. 134) de manière caractéristique " qu'un certain nombre de regimes formellement parlementaires (Italie, $\mathrm{H}$ ollande) fonctionnent en réalité comme des régimes d'assemblée, parce que le jeu de partis multiples y paralyse l'action du gouvernement ».

107. B. Jeanneau le reconnaît explicitement, puisqu'il affirme (op. cit., p. 134) que la notion de " régime d'assemblée " " conserve son intérêt, du moins comme terme de référence ou de contre modele dans l'éventail des formes de gouvernement ». C'est que sel on lui, « il n'existe pas d'autre formule pour désigner cette dépendance de fait du pouvoir Exécutif »; toujours selon lui, la preuve de la nécessité de conserver cette catégorie, pouvait se trouver dans le fait que Michel Debré « pourtant très averti de la science constitutionnelle, (utilisait) couramment l'expression "régime d'assemblée " quand il (voulait) mettre en garde contre un retour à la situation qui a été celle de la France sous toute la IV e République » (ibid.). Ces quelques lignes illustrent parfaitement l'usage justificatif ou idéologique qui est généralement fait de ce concept.

108. On peut ainsi supposer que le concept de "régime d'assemblée » est plus ou moins utilisé en fonction des nécessités doctrinales de l'époque concernée - même si une recherche précise reste à mener. Très utilisé lorsque la France vivait sous un régime parlementaire, et surtout en période de fortes critiques a son encontre (I'ouvrage éponyme de P. Bastid date de 1956), il perd de son utilité lorsque le régime français n'est plus (majoritai rement) considéré comme tel.

109. P. Pactet, op. cit., n. 1, p. 142. C. Leclercq, op. cit., p. 218.

110. B. Chantebout, "Le regime parlementaire moniste, gouvernement d'assemblée », op. cit., p. 43 à 61 .

111. On peut faire remarquer que cette position, en tant que critique radicale du régime parlementaire, aboutit à plaider pour le seul régime politique dès lors disponible : le régime présidentiel. La conclusion de l'article de B. Chantebout est à cet égard edifiante : « en fait II n'existe plus aujourd'hui de juste milieu entre la confusion et la séparation des pouvoirs: ou bien l'on accepte le régime parlementaire moniste dans toute sa réalité de régime d'assemblée, ou bien l'on institue un régime réellement présidentiel, avec l'élection directe du chef de l'État par le peuple, mais aussi avec une séparation réelle des pouvoirs, exclusive de toute responsabilité de l'Exécutif devant les Chambres et de tout moyen de pression excessif sur ces dernières 》 ( $B$. Chantebout, "Le régime parlementaire moniste, gouvernement d'assemblée », op. cit., p. 61).

112. "Le régime d'assemblée est al ors "la dégénérescence normale du régime parlementaire" »(C. Debbasch, op. cit., p. 156). 
Mais par l'usage de ce « spectre », les auteurs peuvent égal ement, et en fait surtout, justifier ou critiquer des règles ou des institutions pré cises - celles qui, précisément, sont annoncées comme les critères distinctifs du "régime d'assemblée ». Soutenir en effet que l'existence (ou l'absence) de telle ou telle disposition constitutionnelle caractérise ce type de régimes équivaut en fait à légitimer l'existence (ou l'absence) de la règle opposée. C'est cela qui explique en grande partie que les divergences doctrinal es quant aux définitions du régime d'assemblée aient si longtemps persisté: le choix de ses critères distinctifs est resté un moyen très pratique de justifier ou de critiquer des institutions ou des règles très diverses (l'existence et les pouvoirs d'une seconde chambre, le statut et les prérogatives du chef de l'État, l'efficacité du droit de dissolution, la responsabilité politique des ministres et leur fonction "gouvernementale », etc. $\left.{ }^{113}\right)$. Les spécificités attribuées au "régime d'assemblée », les critères retenus pour le définir, reflètent ainsi la fonction assignée à ce concept par chacun des auteurs.

Si la notion de « régime d'assemblée » a longtemps joué le rôle d'un véritable " spectre », celui-ci n'est plus guère brandi aujourd'hui que par une sorte d'habitude. Aussi évoque-t-on de plus en plus sa disparition prochaine. Cette déchéance annoncée tient au fait que, pour de multiples raisons, le « régime d'assemblée » a cessé de faire peur.

Le succès du concept de "régime d'assemblée » s'est en réalité prolongé aussi longtemps qu'aura perduré le contexte de méfiance envers la toute-puissance parlementaire, qui avait favorisé son éclosion au tournant du siècle dernier. Cette défiance s'est, on le sait, dével oppée tout au long des III et IV e Républiques jusqu'à I'adoption de la Constitution de 1958, qui a précisément été conçue comme un véritable antidote à ce danger. Désormais, le Parlement n'est à l'évidence plus le lieu réel du pouvoir, et I'on sourirait aujourd'hui de la naïveté de celui qui s'alarmerait de son éventuelle omnipotence ${ }^{114}$. L'heure est plutôt, de nos jours, aux tentatives de reval orisation de son rôle, tant il a été amoindri.

La peur a donc aujourd'hui d'autres visages, et les « spectres » que I'on brandit ont changé de nature. L'un d'entre eux, le plus en vogue, est sans conteste celui du " gouvernement des juges ». En effet, les fonctions de plus en plus importantes remplies par les juges (qu'il s'agisse du Conseil Constitutionnel ou des magistrats des ordres administratif et judiciaire) provoquent de nos jours les débats les plus passionnés, notamment en raison de leur caractère non-électif. Aussi les auteurs se plai-

113. Cf. supra.

114. C'est pourquoi l'argument sel on lequel l'adoption du quinquennat risquerait d'entraîner la France vers le régime d'assemblée nous paraît si suranné (cf. supra). 
sent-ils à souligner le danger que représenterait une (pourtant improbable) dérive vers un système dans lequel les juges seraient chargés de gouverner.

Mais si I'on se contente d'observer l'évolution des régimes dits parlementaires, on doit surtout constater que le pouvoir réel est passé des mains des chambres législ atives à celles des organes gouvernementaux. Si concentration des pouvoirs il y a, elle se fait aujourd'hui indéniablement au profit du gouvernement - ou, pour ce qui est de la $V$ e $R$ épublique, du couple Président-Premier ministre. On observe en effet, dans ces systèmes, que l'organe exécutif, maître de l'élaboration des lois (puisque le Parlement ne fait plus office que de chambre d'enregistrement) autant que de leur exécution, ne risque en outre (presque) jamais de voir sa responsabilité politique être mise en jeu par les députés. Sauf le contrôle des juges constitutionnels (qui ne saurait être véritablement politique), il ne reste plus pour s'opposer aux volontés gouvernementales que le poids relatif d'une opinion publique insaisissable, pour autant qu'elle ait jamais véritablement existé.

Telle est sans doute l'objection la plus radicale que I'on puisse aujourd'hui adresser aux régimes parlementai res contemporains. O seraiton dès lors - sous forme de boutade - suggérer aux partisans de ces derniers de créer, en réponse à cette nouvelle critique et à la manière d'Esmein, un «type » inédit de régimes politiques, qui se distinguerait du régime parlementaire par la concentration des pouvoirs qui y serait instituée au profit de l'organe exécutif? 